\title{
Asymptotic boundary estimates for solutions to the $p$-Laplacian with infinite boundary values
}

Ling $\mathrm{Mi}^{1^{*}}$ (B)

"Correspondence: mi-ling@163.com

'School of Mathematics and Statistics, Linyi University, Linyi,

P.R. China

\begin{abstract}
In this paper, by using Karamata regular variation theory and the method of upper and lower solutions, we mainly study the second order expansion of solutions to the following $p$-Laplacian problems: $\Delta_{p} u=b(x) f(u), u>0, x \in \Omega,\left.u\right|_{\partial \Omega}=\infty$, where $\Omega$ is a bounded domain with smooth boundary in $\mathbb{R}^{N}(N \geq 2), p>1, b \in C^{\alpha}(\bar{\Omega})$ which is positive in $\Omega$ and may be vanishing on the boundary. The absorption term $f$ is normalized regularly varying at infinity with index $\sigma>p-1$. The results extend some previous findings of D. Repovš (J. Math. Anal. Appl. 395:78-85, 2012) in a certain sense.
\end{abstract}

Keywords: $p$-Laplacian problems; Second expansion of solutions; Upper and lower solutions; Karamata regular variation theory

\section{Introduction and the main results}

In this paper, we mainly consider the second order expansion of solutions near the boundary to the following boundary blow-up problem:

$$
\Delta_{p} u=b(x) f(u), \quad u>0, x \in \Omega,\left.u\right|_{\partial \Omega}=\infty
$$

where $\Delta_{p} u:=\operatorname{div}\left(|\nabla u|^{p-2} \nabla u\right)$ stands for a $p$-Laplacian operator with $p>1$, the last condition means that $u(x) \rightarrow+\infty$ as $d(x):=\operatorname{dist}(x, \partial \Omega) \rightarrow 0, \Omega$ is a bounded domain with smooth boundary in $\mathbb{R}^{N}(N \geq 2)$, $b$ satisfies

$\left(b_{1}\right) b \in C^{\alpha}(\bar{\Omega})$ for some $\alpha \in(0,1)$ is positive in $\Omega$;

$\left(b_{2}\right)$ there exist $k \in \Lambda, c \in \mathbb{R}$, and $\theta>0$ such that

$$
b(x)=k^{p}(d(x))\left(1+c(d(x))^{\theta}+o\left((d(x))^{\theta}\right) \quad \text { near } \partial \Omega,\right.
$$

where $\Lambda$ denotes the set of all positive non-decreasing functions in $C^{1}\left(0, \delta_{0}\right)$ which satisfy

$$
\left\{\begin{array}{l}
\lim _{t \rightarrow 0^{+}} \frac{K(t)}{k(t)}=0, \quad K(t)=\int_{0}^{t} k(s) d s ; \\
\lim _{t \rightarrow 0^{+}} \frac{d}{d t}\left(\frac{K(t)}{k(t)}\right):=C_{k} \in[0,1],
\end{array}\right.
$$

and $f$ satisfies

(c) The Author(s) 2019. This article is distributed under the terms of the Creative Commons Attribution 4.0 International License (http://creativecommons.org/licenses/by/4.0/), which permits unrestricted use, distribution, and reproduction in any medium, provided you give appropriate credit to the original author(s) and the source, provide a link to the Creative Commons license, and indicate if changes were made. 
$\left(f_{1}\right) f \in C^{1}[0, \infty), f(0)=0, f$ is increasing on $(0, \infty)$;

$\left(f_{2}\right)$ there exist $\sigma>p-1$ and a function $E \in C^{1}\left[S_{0}, \infty\right)$ for $S_{0}$ large enough such that

$$
\frac{f^{\prime}(s) s}{f(s)}:=\sigma+E(s), \quad s \geq S_{0} \text { with } \lim _{s \rightarrow \infty} E(s)=0,
$$

i.e.,

$$
f(s)=c_{0} s^{\sigma} \exp \left(\int_{S_{0}}^{s} \frac{E(v)}{v} d v\right), \quad s \geq S_{0}, c_{0}>0
$$

$\left(f_{3}\right)$ there exists $\eta \leq 0$ such that

$$
\lim _{s \rightarrow \infty} \frac{E^{\prime}(s) s}{E(s)}=\eta
$$

with $E$ as in Condition $\left(f_{2}\right)$.

A local weak solution to problem (1.1) is meant as a function $u \in C(\Omega) \cap W_{\mathrm{Loc}}^{1, p}(\Omega)$ with $u(x) \rightarrow \infty$ as $d(x):=\operatorname{dist}(x, \partial \Omega) \rightarrow 0$ and, for every $D \subset \subset \Omega$, it holds

$$
\int_{D}|\nabla u|^{p-2} \nabla u \nabla \phi d x=\int_{D} b(x) g(u) \phi d x, \quad \forall \phi \in W_{0}^{1, p}(D) .
$$

The investigation of problem (1.1) has a long history. Since the pioneering work of Bieberbach [2], the problem of existence, asymptotic boundary behavior, and uniqueness of solutions to

$$
\Delta u=b(x) f(u), \quad u>0, x \in \Omega,\left.u\right|_{\partial \Omega}=\infty,
$$

has been extensively studied.

For $b(x) \equiv 1$, Keller-Osserman [3, 4] first supplied a necessary and sufficient condition

$$
\int_{1}^{\infty} \frac{d v}{\sqrt{2 F(v)}}<\infty, \quad F(v)=\int_{0}^{v} f(s) d s
$$

for the existence of solutions of problem (1.2).

Loewner and Nirenberg [5] showed that if $f(u)=u^{p_{0}}$ with $p_{0}=\frac{N+2}{N-2}, N>2$, problem (1.2) has a unique solution $u$ satisfying

$$
\lim _{d(x) \rightarrow 0} u(x)(d(x))^{(N-2) / 2}=\left(\frac{N(N-2)}{4}\right)^{(N-2) / 4} .
$$

Bandle and Marcus [6] proved that if $f$ satisfies $\left(f_{1}\right)$ and the condition that

$\left(f_{1}^{\prime}\right)$ there exist $q>0$ and $S_{0} \geq 1$ such that $f(\xi s) \leq \xi^{1+q} f(s)$ for all $\xi \in(0,1)$ and $s \geq S_{0} / \xi$, then, for any solution $u$ of problem (1.1),

$$
\frac{u(x)}{\psi(d(x))} \rightarrow 1 \quad \text { as } d(x) \rightarrow 0,
$$


where $\psi$ satisfies

$$
\int_{\psi(t)}^{\infty} \frac{d v}{\sqrt{2 F(v)}}=t, \quad \forall t>0
$$

Moreover, if $f$ satisfies

$\left(f_{2}^{\prime}\right) f(s) / s$ is increasing in $(0, \infty)$,

problem (1.2) has a unique solution.

It is very worthwhile to point out that Cîrstea and Rădulescu [7-9], Cîrstea and Du [10] introduced the Karamata regular variation theory to study the boundary behavior and uniqueness of solutions for boundary blow-up elliptic problems and obtained a series of rich and significant data about the boundary behavior of solutions.

Recently, by using the Karamata regular variation theory, Zhang et al. [11], Zhang [12], Huang et al. [13, 14], Mi and Liu [15] further studied the second order expansion of the solutions to problem (1.2). They showed that the second term in the boundary asymptotic expansion of solutions $u(x)$ depends on the weight function $b(x)$.

Now, let us return to problem (1.1).

For $b(x) \equiv 1$ on $\Omega$, Gladiali and Porru [16] studied boundary asymptotic behavior of solutions for (1.1) under some conditions on $f$. They showed that if $F(t) t^{1-p}$ is increasing for large $t$, then a solution $u$ to problem (1.1) satisfies

$$
|u(x)-\psi(d(x))|<c d(x) \psi(d(x)) \quad \text { near } \partial \Omega
$$

with

$$
\int_{\psi(t)}^{\infty}(q F(t))^{-1 / p}=t, \quad t>0
$$

Furthermore, they showed that if $F(t) t^{-2 p} \rightarrow \infty$ as $t \rightarrow \infty$, then

$$
\lim _{d(x) \rightarrow 0} u(x)-\psi(d(x)) \rightarrow 0 \quad \text { as } d(x) \rightarrow 0 .
$$

In Mohammed [17], it was shown that problem (1.1) has a local weak solution if $b \in C(\Omega)$ is a positive function for which the problem $\triangle_{p} v=-b(x)$ admits a solution in $W_{0}^{1, p}(\Omega)$. In particular, $b$ is allowed to vanish on the boundary $\partial \Omega$ or $b$ may be unbounded on $\Omega$. Later, Mohammed [18] continued to consider the boundary asymptotic and uniqueness of solutions for problem (1.1).

For the other works on $p$-Laplacian problem, see [1, 19-29] and the references therein. Inspired by the above works, our objective in this paper is to establish the second order expansion of solutions to problem (1.1) under appropriate conditions on weight function $b(x)$ and non-linearity $f$.

To present our main results, we introduce the following subclass for $\Lambda$.

Let $\beta, \varsigma>0$, we define

$$
\begin{aligned}
& \Lambda_{1, \beta}=\left\{k \in \Lambda \text { with } C_{k} \in[0,1], \lim _{t \rightarrow 0^{+}}(-\ln t)^{\beta}\left(\frac{d}{d t}\left(\frac{K(t)}{k(t)}\right)-C_{k}\right)=D_{1 k} \in \mathbb{R}\right\} ; \\
& \Lambda_{2,5}=\left\{k \in \Lambda \text { with } C_{k} \in(0,1], \lim _{t \rightarrow 0^{+}} \frac{1}{t^{5}}\left(\frac{d}{d t}\left(\frac{K(t)}{k(t)}\right)-C_{k}\right)=D_{2 k} \in \mathbb{R}\right\} ;
\end{aligned}
$$




$$
\Lambda_{3, \varsigma}=\left\{k \in \Lambda \text { with } C_{k}=0, \lim _{t \rightarrow 0^{+}} \frac{1}{t \varsigma}\left(\frac{d}{d t}\left(\frac{K(t)}{k(t)}\right)\right)=D_{3 k} \in \mathbb{R}\right\} .
$$

In the sequel, $\beta$ and $\varsigma$ are understood in the above range.

In this paper, we need the following assumptions.

$\left(f_{4}\right)$ If $\eta=0$ in $\left(f_{3}\right)$, there exists $q_{1} \in \mathbb{R}$ such that

$$
\lim _{s \rightarrow \infty}(\ln s)^{\beta} E(s)=q_{1},
$$

where $\beta$ is the parameter used in the definition of $\Lambda_{1, \beta}$;

$\left(f_{5}\right) \eta \leq-\frac{(\sigma+1-p)_{\varsigma}}{p}$ in $\left(\mathrm{f}_{3}\right)$ and $\lim _{s \rightarrow \infty} s^{\frac{(\sigma+1-p)_{\varsigma}}{p}} E(s)=q_{2} \in \mathbb{R}$ if $\eta=-\frac{(\sigma+1-p)_{\varsigma}}{p}$,

where $\zeta$ is the parameter used in the definition of $\Lambda_{2, \varsigma}$;

$\left(f_{6}\right)$ If $\eta=0$ in $\left(f_{3}\right)$, there exists $q_{3} \in \mathbb{R}$ such that

$$
\lim _{s \rightarrow \infty}(\ln s)^{\tau} E(s)=q_{3}
$$

where $\tau=\frac{\varpi}{\varsigma}, \varpi=\min \{\theta, \varsigma\}, \varsigma$ is the parameter used in the definition of $\Lambda_{3, \varsigma}$.

The key of our estimates is the solution to the problem

$$
\int_{\phi(t)}^{\infty} \frac{d v}{(q F(v))^{\frac{1}{p}}}=t, \quad t>0
$$

Here $q$ stands for the Hölder conjugate of $p$.

Our main results are summarized as follows.

Theorem 1.1 Suppose that $f$ satisfies $\left(f_{1}\right)-\left(f_{2}\right), b$ satisfies $\left(b_{1}\right)-\left(b_{2}\right), k \in \Lambda_{1, \beta}$, and one of the following conditions holds:

(i) $f(s)=C s^{\sigma}(\sigma>p-1)$ in a neighborhood of infinity;

(ii) $f$ satisfies $\left(f_{3}\right)$ with $\eta<0$ and $D_{1 k} \neq 0$;

(iii) $f$ satisfies $\left(f_{3}\right)$ with $\eta=0,\left(f_{4}\right)$ holds and $D_{1 k}^{2}+\left(C_{k} q_{1}\right)^{2} \neq 0$.

Then, for the unique solution $u$ of problem (1.1) and all $x$ in a neighborhood of $\partial \Omega$,

$$
u(x)=A_{1} \phi(K(d(x)))\left(1+A_{2}(-\ln (d(x)))^{-\beta}+o\left((-\ln (d(x)))^{-\beta}\right)\right)
$$

where $\phi$ is uniquely determined by (1.7) and

$$
\begin{aligned}
& A_{1}=\left(\frac{C_{k}(\sigma+1-p)+p}{\sigma+1}\right)^{\frac{1}{\sigma-p+1}} ; \\
& A_{2}= \begin{cases}\frac{D_{1 k}}{p+(\sigma+1-p) C_{k}} & \text { if (i) or (ii) holds; } \\
\frac{D_{1 k}}{p+(\sigma+1-p) C_{k}}-\frac{q_{1} \xi_{1}(\sigma+1)\left(\frac{p\left(1-C_{k}\right)}{(\sigma+1)(\sigma+1+\eta)}+A_{1}^{\sigma-p+1} \ln A_{1}\right)}{(\sigma+1-p)\left(p+(\sigma+1-p) C_{k}\right)} & \text { if (iii) holds, }\end{cases}
\end{aligned}
$$

where $\xi_{1}=p^{-\beta}\left((p-1-\sigma) C_{k}\right)^{\beta}$.

Theorem 1.2 Let $f$ satisfy $\left(f_{1}\right)-\left(f_{2}\right)$, b satisfy $\left(b_{1}\right)-\left(b_{2}\right), \varsigma<\frac{p(1+\sigma)}{\sigma+1-p}$, and $\varpi<\frac{p}{(\sigma+1-p) C_{k}}$. Suppose $k \in \Lambda_{2, \varsigma}$ with $C_{k} \in(0,1)$, and one of the following conditions holds:

(i) $f(s)=C s^{\sigma}(\sigma>p-1)$ in a neighborhood of infinity;

(ii) $f$ satisfies $\left(f_{3}\right)$ and $\left(f_{5}\right)$. 
Then, for the unique solution u of problem (1.1) and all $x$ in a neighborhood of $\partial \Omega$,

$$
u(x)=A_{1} \phi(K(d(x)))\left(1+A_{3}(d(x))^{\sigma}+o\left((d(x))^{\sigma}\right)\right)
$$

where $\phi$ is uniquely determined by (1.7), $\varpi=\min \{\theta, \varsigma\}, A_{1}$ is in Theorem 1.1 and

$$
A_{3}=-\frac{p(\sigma+1-p) D_{2 k} \text { Heaviside }(\theta-\varsigma)-c\left(p+(\sigma+1-p) C_{k}\right) \text { Heaviside }(\varsigma-\theta)}{(\sigma+1-p)\left(C_{k}(\sigma+1-p)\left(\varpi(\varpi+1) C_{k}-p\left(1+\varpi C_{k}\right)\right)-p^{2}\left(1+\varpi C_{k}\right)\right)} .
$$

Theorem 1.3 Let $f$ satisfy $\left(f_{1}\right)-\left(f_{2}\right), b$ satisfy $\left(b_{1}\right)-\left(b_{2}\right)$. Suppose that $k \in \Lambda_{3,5}$ and one of the following conditions holds:

(i) $f(s)=C s^{\sigma}(\sigma>p-1)$ in a neighborhood of infinity;

(ii) $f$ satisfies $\left(f_{3}\right)$ with $\eta<0$;

(iii) $f$ satisfies $\left(f_{3}\right)$ with $\eta=0$ and $\left(f_{6}\right)$ holds.

Then, for the unique solution $u$ of problem (1.1) and all $x$ in a neighborhood of $\partial \Omega$,

$$
u(x)=A_{1} \phi(K(d(x)))\left(1+A_{4}(d(x))^{\varpi}+o\left((d(x))^{\varpi}\right)\right)
$$

where $\phi$ is uniquely determined by (1.7), $A_{1}=\left(\frac{p}{\sigma+1}\right)^{\frac{1}{\sigma-p+1}}$, and

$$
A_{4}= \begin{cases}\frac{1}{p} D_{3 k} \operatorname{Heaviside}(\theta-\varsigma)+\frac{c}{\sigma+1-p} \operatorname{Heaviside}(\varsigma-\theta):=A_{5} & \text { if (i) or (ii) holds; } \\ A_{5}-\xi_{2} q_{3}\left(\frac{1}{(\sigma+1+\eta)(\sigma+1-p)}+\frac{\ln \frac{p}{p+1}}{(\sigma+1-p)^{2}}\right) & \text { if (iii) holds, }\end{cases}
$$

where $\xi_{2}=(2(\varsigma+1))^{-\tau}\left((p-1) \varsigma D_{3 k}\right)^{\tau}$.

Remark 1.1 For the existence of solutions for problem (1.1), see Mohammed [17]. For the uniqueness of solutions for problem (1.1), see Mohammed [18].

Remark 1.2 In Theorem 1.1, suppose (i) or (ii) holds. When $C_{k} \in[0,1]$, the constant $A_{2}$ is defined by the same formula:

$$
A_{2}=\frac{D_{1 k}}{p+(\sigma+1-p) C_{k}} .
$$

Remark 1.3 When $k \in \Lambda_{1, \beta}, \Lambda_{2, \varsigma}$, or $\Lambda_{3,5}$, the second order expansion of $u$ (see (1.8)(1.10)) only involves the distance $d(x)$.

Remark 1.4 Some basic examples of functions for the function $E$ are:

(i) when $f(s)=C s^{\sigma}(\ln s)^{\beta}\left(\sigma>p-1, s \geq S_{0}\right), E(s)=\beta(\ln s)^{-1}$;

(ii) when $f(s)=C s^{\sigma} \exp \left((\ln s)^{\beta}\right)\left(\sigma>p-1, \beta<1, s \geq S_{0}\right), E(s)=\beta(\ln s)^{\beta-1}$;

(iii) when $f(s)=C s^{\sigma} \exp \left(s^{\beta}\right)\left(\sigma>p-1, \beta \leq 0, s \geq S_{0}\right), E(s)=\beta s^{\beta}$.

The outline of this paper is as follows. In Sect. 2, we give some preliminary results of regularly varying functions. In Sect. 3 , we give some auxiliary results that will be used in the next sections. The proofs of Theorems 1.1-1.3 are in the next sections. 


\section{Some properties of regularly varying function}

The Karamata regular variation theory was first introduced and established by Karamata in 1930 and is a basic tool in stochastic processes (see [30-32]). In this section, we present some bases of Karamata regular variation theory.

A positive measurable function $f$ defined on $[a, \infty)$, for some $a>0$, is called regularly varying at infinity with index $\rho$, written $f \in R V_{\rho}$, if for each $\xi>0$ and some $\rho \in \mathbb{R}$,

$$
\lim _{s \rightarrow \infty} \frac{f(\xi s)}{f(s)}=\xi^{\rho} .
$$

In particular, when $\rho=0, f$ is called slowly varying at infinity.

Clearly, if $f \in R V_{\rho}$, then $L(s):=f(s) / s^{\rho}$ is slowly varying at infinity.

We also see that a positive measurable function $h$ defined on $(0, a)$ for some $a>0$ is regularly varying at zero with index $\rho$ (write $h \in R V Z_{\rho}$ ) if $t \rightarrow h(1 / t)$ belongs to $R V_{-\rho}$.

Proposition 2.1 (Uniform convergence theorem) If $f \in R V_{\rho}$, then (2.1) holds uniformly for $\xi \in\left[c_{1}, c_{2}\right]$ with $0<c_{1}<c_{2}$.

Proposition 2.2 (Representation theorem) A function L is slowly varying at infinity if and only if it may be written in the form

$$
L(s)=\varphi(s) \exp \left(\int_{a_{1}}^{s} \frac{y(v)}{v} d v\right), \quad s \geq a_{1},
$$

for some $a_{1} \geq a$, where the functions $\varphi$ and $y$ are measurable and for $s \rightarrow \infty, y(s) \rightarrow 0$ and $\varphi(s) \rightarrow c_{0}$, with $c_{0}>0$.

We call that

$$
\hat{L}(s)=c_{0} \exp \left(\int_{a_{1}}^{s} \frac{y(v)}{v} d v\right), \quad s \geq a_{1}
$$

is normalized slowly varying at infinity and

$$
f(s)=s^{\rho} \hat{L}(s), \quad s \geq a_{1},
$$

is normalized regularly varying at infinity with index $\rho$ (and write $f \in \mathrm{NRV}_{\rho}$ ).

Similarly, $g$ is called normalized regularly varying at zero with index $\rho$, written $g \in$ $\mathrm{NRVZ}_{\rho}$, if $t \rightarrow g(1 / t)$ belongs to $\mathrm{NRV}_{-\rho}$.

A function $f \in R V_{\rho}$ belongs to $\mathrm{NRV}_{\rho}$ if and only if

$$
f \in C^{1}\left[a_{1}, \infty\right) \text { for some } a_{1}>0 \text { and } \lim _{s \rightarrow \infty} \frac{s f^{\prime}(s)}{f(s)}=\rho .
$$

Proposition 2.3 If functions $L, L_{1}$ are slowly varying at infinity, then

(i) $L^{\rho}$ for every $\rho \in \mathbb{R}, c_{1} L+c_{2} L_{1}\left(c_{1} \geq 0, c_{2} \geq 0\right.$ with $\left.c_{1}+c_{2}>0\right), L \circ L_{1}\left(\right.$ if $L_{1}(t) \rightarrow \infty$ as $t \rightarrow \infty)$ are also slowly varying at infinity.

(ii) For every $\rho>0$ and $t \rightarrow \infty$,

$$
t^{\rho} L(t) \rightarrow \infty, \quad t^{-\rho} L(t) \rightarrow 0 .
$$


(iii) For $\rho \in \mathbb{R}$ and $t \rightarrow \infty, \ln (L(t)) / \ln t \rightarrow 0$ and $\ln \left(t^{\rho} L(t)\right) / \ln t \rightarrow \rho$.

\section{Proposition 2.4}

(i) If $g_{1} \in R V Z_{\rho_{1}}, g_{2} \in R V Z_{\rho_{2}}$ with $\lim _{t \rightarrow 0^{+}} g_{2}(t)=0$, then $g_{1} \circ g_{2} \in R V Z_{\rho_{1} \rho_{2}}$.

(ii) If $g \in R V Z_{\rho}$, then $g^{\alpha} \in R V Z_{\rho \alpha}$ for every $\alpha \in \mathbb{R}$.

Proposition 2.5 (Asymptotic behavior) If a function $L$ is slowly varying at infinity, then for $a \geq 0$ and $t \rightarrow \infty$,

(i) $\int_{a}^{t} s^{\rho} L(s) d s \cong(\rho+1)^{-1} t^{1+\rho} L(t)$ for $\rho>-1$;

(ii) $\int_{t}^{\infty} s^{\rho} L(s) d s \cong(-\rho-1)^{-1} t^{1+\rho} L(t)$ for $\rho<-1$.

\section{Auxiliary results}

In this section, we collect some useful results.

Lemma 3.1 (Lemma 3.1 in [15]) Let $k \in \Lambda$. Then

(i) $\lim _{t \rightarrow 0^{+}} \frac{K(t)}{k(t)}=0, \lim _{t \rightarrow 0^{+}} \frac{K(t)}{t k(t)}=C_{k}$;

(ii) $\lim _{t \rightarrow 0^{+}} \frac{K(t) k^{\prime}(t)}{k^{2}(t)}=1-C_{k}$;

(iii) when $k \in \Lambda_{1, \beta}$,

$$
\lim _{t \rightarrow 0^{+}}(-\ln t)^{\beta}\left(\frac{K(t) k^{\prime}(t)}{k^{2}(t)}-\left(1-C_{k}\right)\right)=-D_{1 k}
$$

(iv) when $k \in \Lambda_{2,5}$,

$$
\lim _{t \rightarrow 0^{+}} \frac{1}{t^{5}}\left(\frac{K(t) k^{\prime}(t)}{k^{2}(t)}-\left(1-C_{k}\right)\right)=-D_{2 k} ;
$$

(v) when $k \in \Lambda_{3,5}$,

$$
\lim _{t \rightarrow 0^{+}} \frac{1}{t^{5}}\left(\frac{K(t) k^{\prime}(t)}{k^{2}(t)}-1\right)=-D_{3 k} .
$$

Lemma 3.2 Iff satisfies $\left(f_{1}\right)-\left(f_{2}\right)$, then

(i) $f$ satisfies the generalized Keller-Osserman condition

$$
\int_{1}^{\infty}(q F(t))^{-1 / p}<+\infty, \quad F(t)=\int_{0}^{t} f(s) d s
$$

(ii)

$$
\lim _{s \rightarrow \infty} f^{\prime}(s) \int_{s}^{\infty} \frac{d v}{f(v)}=\frac{\sigma}{\sigma-1}
$$

and

$$
\lim _{s \rightarrow \infty} \frac{f(s) \int_{s}^{\infty} \frac{d v}{f(v)}}{s}=\frac{1}{\sigma-1} ;
$$

(iii) there exists $S_{0}>0$ such that $\frac{f(s)}{s^{m}}$ is increasing in $\left[S_{0}, \infty\right)$, where $m \in(1, \sigma)$; 
(iv)

$$
\lim _{s \rightarrow \infty} \frac{s f(s)}{F(s)}=\sigma+1
$$

(v)

$$
\lim _{s \rightarrow \infty} \frac{s(F(s))^{-\frac{1}{p}}}{\int_{s}^{\infty}(F(t))^{-\frac{1}{p}} d t}=\frac{\sigma+1-p}{p} .
$$

Proof By $f \in \mathrm{NRV}_{\sigma}$ with $\sigma>p-1$, we see that $f(s)=c_{0} s^{\sigma} \hat{L}(s)$ in $\left[S_{0}, \infty\right)$, where $\hat{L}$ is normalized slowly varying at infinity and $c_{0}>0$. Let $\sigma_{1} \in(p-1, \sigma)$. It follows by Proposition 2.3(ii) that

$$
\lim _{s \rightarrow \infty} s^{\sigma-\sigma_{1}} \hat{L}(s)=\infty
$$

Then there exists $S_{1}>S_{0}$ such that

$$
c_{0} s^{\sigma-\sigma_{1}} \hat{L}(s)>1, \quad \forall s \geq S_{1} \text {, i.e., } f(s) \geq s^{\sigma_{1}}, \forall s \geq S_{1}
$$

and there exists $S_{2}>S_{1}$ such that

$$
F(s) \geq \frac{s^{\sigma_{1}+1}}{\sigma_{1}+1}, \quad \forall s \geq S_{2}
$$

So, (i) holds.

(ii) By $\left(f_{2}\right)$ and Proposition 2.5(ii), we obtain that

$$
\begin{aligned}
\lim _{s \rightarrow \infty} f^{\prime}(s) \int_{s}^{\infty} \frac{d v}{f(v)}= & \lim _{s \rightarrow \infty} \frac{s f^{\prime}(s)}{f(s)} \lim _{s \rightarrow \infty} \frac{f(s)}{s} \int_{s}^{\infty} \frac{d v}{f(v)} \\
& =\sigma \lim _{s \rightarrow \infty} s^{\sigma-1} \hat{L}(s) \int_{s}^{\infty} v^{-\sigma}(\hat{L}(v))^{-1} d v \\
& =\frac{\sigma}{\sigma-1} .
\end{aligned}
$$

It follows by l'Hospital's rule that

$$
\lim _{s \rightarrow \infty} \frac{f(s) \int_{s}^{\infty} \frac{d v}{f(v)}}{s}=\lim _{s \rightarrow \infty} f^{\prime}(s) \int_{s}^{\infty} \frac{d v}{f(v)}-1=\frac{1}{\sigma-1}
$$

(iii) By the choice of $m$ and (ii), one can see that

$$
\lim _{s \rightarrow \infty}\left(f^{\prime}(s)-m \frac{f(s)}{s}\right) \int_{s}^{\infty} \frac{d v}{f(v)}=\frac{\sigma-m}{\sigma-1}>0 .
$$

Then there exists $S_{0}>0$ such that $\left(\frac{f(s)}{s^{m}}\right)^{\prime}=s^{-m}\left(f^{\prime}(s)-m \frac{f(s)}{s}\right)>0, \forall s \geq S_{0}$, i.e., $f(s) / s^{m}$ is increasing on $\left[S_{0}, \infty\right)$. 
(iv) By $\left(f_{2}\right)$ and Proposition 2.5(i), we obtain that

$$
\begin{aligned}
\lim _{s \rightarrow \infty} \frac{s f(s)}{F(s)} & =\lim _{s \rightarrow \infty} \frac{s^{\sigma+1} \hat{L}(s)}{\int_{0}^{s} v^{\sigma} \hat{L}(\nu) d v} \\
& =\lim _{s \rightarrow \infty} \frac{s^{\sigma+1} \hat{L}(s)}{(\sigma+1)^{-1} s^{1+\sigma} \hat{L}(s)} \\
& =\sigma+1 .
\end{aligned}
$$

(v) It follows by (iv) that $F \in \mathrm{NRV}_{\sigma+1}$ with $\sigma+1>p$. By Proposition 2.4(ii), we have $F^{-\frac{1}{p}} \in \mathrm{NRV}_{-\frac{\sigma+1}{p}}$. Hence, by Proposition 2.4(ii), we obtain

$$
\begin{aligned}
\lim _{s \rightarrow \infty} \frac{s(F(s))^{-\frac{1}{p}}}{\int_{s}^{\infty}(F(t))^{-\frac{1}{p}} d t} & =\lim _{s \rightarrow \infty} \frac{s^{-\frac{\sigma+1}{p}+1} \hat{L}(s)}{\int_{s}^{\infty} v^{-\frac{\sigma+1}{p}} \hat{L}(\nu) d v} \\
& =\lim _{s \rightarrow \infty} \frac{s^{-\frac{\sigma+1}{p}+1} \hat{L}(s)}{\left(\frac{\sigma+1}{p}-1\right)^{-1} s^{-\frac{\sigma+1}{p}+1} \hat{L}(s)} \\
& =\frac{\sigma+1-p}{p} .
\end{aligned}
$$

Lemma 3.3 Let $f$ satisfy $\left(f_{1}\right)-\left(f_{3}\right)$, and let $\left(f_{4}\right)$ hold, then

(i)

$$
\lim _{s \rightarrow \infty}(\ln s)^{\beta}\left(\frac{F(s)}{s f(s)}-\frac{1}{\sigma+1}\right)=\sigma_{1},
$$

where

$$
\sigma_{1}= \begin{cases}0 & \text { if } \eta<0 \\ -\frac{q_{1}}{(\sigma+1)(\sigma+1+\eta)} & \text { if } \eta=0\end{cases}
$$

(ii)

$$
\lim _{s \rightarrow \infty}(\ln s)^{\beta}\left(\frac{s(F(s))^{-\frac{1}{p}}}{\int_{s}^{\infty}(F(t))^{-\frac{1}{p}} d t}-\frac{\sigma+1-p}{p}\right)=\sigma_{2},
$$

where

$$
\sigma_{2}= \begin{cases}0 & \text { if } \eta<0 \\ \frac{q_{1}(\sigma+1)}{p(\eta+\sigma+1)} & \text { if } \eta=0\end{cases}
$$

(iii)

$$
\lim _{s \rightarrow \infty}(\ln s)^{\beta}\left(\frac{(F(s))^{1-\frac{1}{p}}}{f(s) \int_{s}^{\infty}(F(t))^{-\frac{1}{p}} d t}+\frac{p-\sigma-1}{p(\sigma+1)}\right)=\sigma_{3}
$$


where

$$
\sigma_{3}= \begin{cases}0 & \text { if } \eta<0, \\ \frac{q_{1}}{(\sigma+1)(\sigma+1+\eta)} & \text { if } \eta=0\end{cases}
$$

(iv)

$$
\lim _{s \rightarrow \infty}(\ln s)^{\beta}\left(\frac{f\left(A_{1} s\right)}{A_{1}^{p-1} f(s)}-A_{1}^{\sigma-p+1}\right)=\sigma_{4},
$$

where

$$
\sigma_{4}= \begin{cases}0 & \text { if } \eta<0 \\ q_{1} A_{1}^{\sigma-p+1} \ln A_{1} & \text { if } \eta=0\end{cases}
$$

Proof (i) It follows by $\left(f_{2}\right)$ that

$$
s f^{\prime}(s)=\sigma f(s)+E(s) f(s), \quad s \in\left[S_{0}, \infty\right) .
$$

Integrating it from $S_{0}$ to $s$ and integrating by parts, we obtain that

$$
s f(s)=(\sigma+1) F(s)+\int_{S_{0}}^{s} E(v) f(v) d v+c, \quad s \in\left[S_{0}, \infty\right),
$$

i.e.,

$$
\frac{F(s)}{s f(s)}-\frac{1}{\sigma+1}=-\frac{E(s)}{\sigma+1} \frac{\int_{s_{0}}^{s} f(v) E(v) d v}{s f(s) E(s)}-\frac{c}{(\sigma+1) s f(s)},
$$

where $c$ is a constant.

Since $f \in \mathrm{NRV}_{\sigma}$ with $\sigma>p-1$, we obtain by Propositions 2.5 and 2.3(ii) that

$$
\lim _{s \rightarrow \infty} \frac{\int_{S_{0}}^{s} f(v) E(v) d v}{s f(s) E(s)}=\frac{1}{\sigma+1+\eta} \quad \text { and } \quad \lim _{s \rightarrow \infty} s f(s)(\ln s)^{-\beta}=\infty .
$$

Thus (ii) follows by (3.2).

(ii) By (3.1), it follows that

$$
\frac{t f(t)}{F(t)}=(\sigma+1)+\frac{\int_{S_{0}}^{t} E(s) f(s) d s}{F(t)}+\frac{c}{F(t)}, \quad t \in\left[S_{0}, \infty\right) .
$$

Besides, by a simple calculation, it leads to

$$
\frac{t \frac{d}{d t}\left((F(t))^{-\frac{1}{p}}\right)}{(F(t))^{-\frac{1}{p}}}=-\frac{t f(t)}{p F(t)}=-\frac{\sigma+1}{p}-\frac{\int_{S_{0}}^{t} E(s) f(s) d s}{p F(t)}-\frac{c}{p F(t)}, \quad t \in\left[S_{0}, \infty\right),
$$

i.e.,

$$
t \frac{d}{d t}\left((F(t))^{-\frac{1}{p}}\right)=-\frac{\sigma+1}{p}(F(t))^{-\frac{1}{p}}-\frac{\int_{S_{0}}^{t} E(v) f(v) d v}{p(F(t))^{1+\frac{1}{p}}}-\frac{c}{p(F(t))^{1+\frac{1}{p}}}, \quad t \in\left[S_{0}, \infty\right) .
$$


Since $f \in \mathrm{NRV}_{\sigma}$ with $\sigma>p-1$, by Proposition 2.3(ii), we know $\lim _{s \rightarrow \infty} \frac{s}{(F(s))^{\frac{1}{p}}}=0$.

Hence, integrating (3.6) from $s$ to $\infty$ and integrating by parts, we derive that

$$
\begin{aligned}
s(F(s))^{-\frac{1}{p}}= & \frac{\sigma+1-p}{p} \int_{s}^{\infty} \frac{d t}{(F(t))^{\frac{1}{p}}}+\int_{s}^{\infty} \frac{\int_{S_{0}}^{t} E(v) f(v) d v}{p(F(t))^{1+\frac{1}{p}}} d t \\
& +\int_{s}^{\infty} \frac{c d t}{p(F(t))^{1+\frac{1}{p}}}, \quad s \geq S_{0},
\end{aligned}
$$

i.e.,

$$
\frac{s(F(s))^{-\frac{1}{p}}}{\int_{s}^{\infty} \frac{d t}{(F(t))^{\frac{1}{p}}}}-\frac{\sigma+1-p}{p}=\frac{\int_{s}^{\infty} \frac{\int_{S_{0}}^{t} E(v) f(v) d v}{p(F(t))^{1+\frac{1}{p}}} d t+\int_{s}^{\infty} \frac{c d t}{p(F(t))^{1+\frac{1}{p}}}}{\int_{s}^{\infty} \frac{d t}{(F(t))^{\frac{1}{p}}}}, \quad s \geq S_{0}
$$

By l'Hospital's rule it follows that

$$
\begin{gathered}
\lim _{s \rightarrow \infty}(\ln s)^{\beta} \frac{\int_{s}^{\infty} \frac{\int_{S_{0}}^{t} E(v) f(v) d v}{p(F(t))^{1+\frac{1}{p}}} d t+\int_{s}^{\infty} \frac{c d t}{p(F(t))^{1+\frac{1}{p}}}}{\int_{s}^{\infty} \frac{d t}{(F(t))^{\frac{1}{p}}}} \\
=\frac{1}{p} \lim _{s \rightarrow \infty} \frac{(\ln s)^{\beta} E(s) \frac{\int_{S_{0}}^{t} E(v) f(v) d v}{s E(s) f(s)} \frac{s f(s)}{F(s)}+c \frac{(\ln s)^{\beta}}{F(s)}}{1+(s \ln s)^{-1} \beta(F(t))^{\frac{1}{p}} \int_{s}^{\infty} \frac{d t}{(F(t))^{\frac{1}{p}}}}=\sigma_{2} .
\end{gathered}
$$

(iii) By a simple calculation, we have

$$
\begin{aligned}
& \lim _{s \rightarrow \infty}(\ln s)^{\beta}\left(\frac{(F(s))^{1-\frac{1}{p}}}{f(s) \int_{s}^{\infty}(F(t))^{-\frac{1}{p}} d t}+\frac{p-\sigma-1}{p(\sigma+1)}\right) \\
& =\lim _{s \rightarrow \infty}(\ln s)^{\beta}\left(\frac{F(s)}{s f(s)} \frac{s(F(s))^{-\frac{1}{p}}}{\int_{s}^{\infty}(F(t))^{-\frac{1}{p}} d t}+\frac{p-\sigma-1}{p(\sigma+1)}\right) \\
& =\lim _{s \rightarrow \infty}(\ln s)^{\beta}\left(\left(\frac{F(s)}{s f(s)}-\frac{1}{\sigma+1}\right)\left(\frac{s(F(s))^{-\frac{1}{p}}}{\int_{s}^{\infty}(F(t))^{-\frac{1}{p}} d t}+\frac{p-\sigma-1}{p}\right)\right. \\
& \left.\quad-\frac{p-\sigma-1}{p}\left(\frac{F(s)}{s f(s)}-\frac{1}{\sigma+1}\right)+\frac{1}{\sigma+1}\left(\frac{s(F(s))^{-\frac{1}{p}}}{\int_{s}^{\infty}(F(t))^{-\frac{1}{p}} d t}+\frac{p-\sigma-1}{p}\right)\right) .
\end{aligned}
$$

Hence, by (i)-(ii), we get

$$
\lim _{s \rightarrow \infty}(\ln s)^{\beta}\left(\frac{(F(s))^{1-\frac{1}{p}}}{f(s) \int_{s}^{\infty}(F(t))^{-\frac{1}{p}} d t}+\frac{p-\sigma-1}{p(\sigma+1)}\right)=\sigma_{3} .
$$

(iv) When $A_{1}=1$, the result is obvious. Now let $A_{1} \neq 1$. By $\left(f_{2}\right)$, we see that

$$
\frac{f\left(A_{1} s\right)}{A_{1}^{p-1} f(s)}-A_{1}^{\sigma-p+1}=A_{1}^{\sigma-p+1}\left(\exp \left(\int_{s}^{A_{1} s} \frac{E(v)}{v} d v\right)-1\right) .
$$


By Proposition 2.1 and $\left(f_{3}\right)$, we know

$$
\lim _{s \rightarrow \infty} \frac{E(s u)}{u}=0 \text { and } \lim _{s \rightarrow \infty} \frac{E(s u)}{E(s) u}=u^{\eta-1}
$$

uniformly with respect to $s \in\left[1, A_{1}\right]$ or $s \in\left[A_{1}, 1\right]$.

So,

$$
\lim _{s \rightarrow \infty} \int_{s}^{A_{1} s} \frac{E(v)}{v} d v=\lim _{s \rightarrow \infty} \int_{1}^{A_{1}} \frac{E(s u)}{u} d u=0
$$

and

$$
\lim _{s \rightarrow \infty} \int_{1}^{A_{1}} \frac{E(s u)}{E(s) u} d u=\int_{1}^{A_{1}} u^{\eta-1} d u=\kappa
$$

where

$$
\kappa= \begin{cases}\frac{1}{\eta}\left(A_{1}^{\eta}-1\right) & \text { if } \eta<0 \\ \ln A_{1} & \text { if } \eta=0\end{cases}
$$

Since $e^{r}-1 \cong r$ as $r \rightarrow 0$, we obtain

$$
\frac{f\left(A_{1} s\right)}{A_{1}^{p-1} f(s)}-A_{1}^{\sigma-p+1} \cong A_{1}^{\sigma-p+1} \int_{s}^{A_{1} s} \frac{E(\nu)}{\nu} d \nu, \quad \text { as } s \rightarrow \infty .
$$

Hence,

$$
\begin{aligned}
& \lim _{s \rightarrow \infty}(\ln s)^{\beta}\left(\frac{f\left(A_{1} s\right)}{A_{1}^{p-1} f(s)}-A_{1}^{\sigma-p+1}\right) \\
& \quad=A_{1}^{\sigma-p+1} \lim _{s \rightarrow \infty}(\ln s)^{\beta} E(s) \lim _{s \rightarrow \infty} \int_{1}^{A_{1}} \frac{E(s u)}{E(s) u} d u=\sigma_{4} .
\end{aligned}
$$

Lemma 3.4 Let $f$ satisfy $\left(f_{1}\right)-\left(f_{3}\right)$, and let $\left(f_{5}\right)$ hold, then

(i)

$$
\lim _{s \rightarrow \infty} s^{\frac{\sigma+1-p}{p}} s\left(\frac{F(s)}{s f(s)}-\frac{1}{\sigma+1}\right)=\kappa_{1},
$$

where

$$
\kappa_{1}= \begin{cases}0 & \text { if } \frac{\sigma+1-p}{p} \varsigma+\eta<0, \\ -\frac{q_{2}}{(\sigma+1)(\sigma+1+\eta)} & \text { if } \frac{\sigma+1-p}{p} \varsigma+\eta=0\end{cases}
$$

(ii)

$$
\lim _{s \rightarrow \infty} s^{\frac{\sigma+1-p}{p}} \varsigma\left(\frac{s(F(s))^{-\frac{1}{p}}}{\int_{s}^{\infty}(F(t))^{-\frac{1}{p}} d t}-\frac{\sigma+1-p}{p}\right)=\kappa_{2},
$$


where

$$
\kappa_{2}= \begin{cases}0 & \text { if } \frac{\sigma+1-p}{p} \varsigma+\eta<0, \\ \frac{(\sigma+1) q_{2}}{p(1+\zeta)(\sigma+1+\eta)} & \text { if } \frac{\sigma+1-p}{p} \varsigma+\eta=0\end{cases}
$$

(iii)

$$
\lim _{s \rightarrow \infty} s^{\frac{\sigma+1-p}{p}} s\left(\frac{(F(s))^{1-\frac{1}{p}}}{f(s) \int_{s}^{\infty}(F(t))^{-\frac{1}{p}} d t}+\frac{p-\sigma-1}{p(\sigma+1)}\right)=\kappa_{3},
$$

where

$$
\kappa_{3}= \begin{cases}0 & \text { if } \frac{\sigma+1-p}{p} \varsigma+\eta<0, \\ \frac{q_{2}(p-\varsigma(\sigma+1-p))}{p(\sigma+1)(\sigma+1+\eta)(1+\varsigma)} & \text { if } \frac{\sigma+1-p}{p} \varsigma+\eta=0\end{cases}
$$

(iv)

$$
\lim _{s \rightarrow \infty} s^{\frac{\sigma+1-p}{p}} \varsigma\left(\frac{f\left(A_{1} s\right)}{A_{1}^{p-1} f(s)}-A_{1}^{\sigma-p+1}\right)=\kappa_{4}
$$

where

$$
\kappa_{4}= \begin{cases}0 & \text { if } \frac{\sigma+1-p}{p} \varsigma+\eta<0, \\ \frac{q_{2}}{\eta} A_{1}^{p-1}\left(A_{1}^{\eta}-1\right) & \text { if } \frac{\sigma+1-p}{p} \varsigma+\eta=0 .\end{cases}
$$

Proof (i) By (3.2), we obtain

$$
\begin{aligned}
& \lim _{s \rightarrow \infty} s^{\frac{\sigma+1-p}{p}} s\left(\frac{F(t)}{t f(t)}-\frac{1}{\sigma+1}\right) \\
& =-\lim _{s \rightarrow \infty} \frac{s^{\frac{\sigma+1-p}{p}} E(s)}{\sigma+1} \lim _{s \rightarrow \infty} \frac{\int_{S_{0}}^{s} f(v) E(v) d v}{s f(s) E(s)}-\lim _{s \rightarrow \infty} \frac{c s^{\frac{\sigma+1-p}{p}} s}{(\sigma+1) s f(s)} .
\end{aligned}
$$

Since $f \in \mathrm{NRV}_{\sigma}$ and $\varsigma<\frac{p(\sigma+1)}{\sigma+1-p}$, by Proposition 2.3(ii), we know $\lim _{s \rightarrow \infty} \frac{\frac{\frac{\sigma+1-p}{p}}{\rho f}}{s f(s)}=0$.

Combining with (3.3), it follows that

$$
\lim _{s \rightarrow \infty} s^{\frac{\sigma+1-p}{p}} s\left(\frac{F(t)}{t f(t)}-\frac{1}{\sigma+1}\right)=\kappa_{1} .
$$

(ii) It follows by (3.7) that

$$
\begin{aligned}
& \lim _{s \rightarrow \infty} s^{\frac{\sigma+1-p}{p}} s\left(\frac{s(F(s))^{-\frac{1}{p}}}{\int_{s}^{\infty} \frac{d t}{(F(t))^{\frac{1}{p}}}}-\frac{\sigma+1-p}{p}\right) \\
& \quad=\lim _{s \rightarrow \infty} s^{\frac{\sigma+1-p}{p}} s \frac{\int_{s}^{\infty} \frac{\int_{S_{0}}^{t} E(v) f(v) d v}{p(F(t))^{1+\frac{1}{p}}} d t+\int_{s}^{\infty} \frac{c d t}{p(F(t))^{1+\frac{1}{p}}}}{\int_{s}^{\infty} \frac{d t}{(F(t))^{\frac{1}{p}}}} .
\end{aligned}
$$


By l'Hospital's rule, it follows that

$$
\begin{aligned}
& \lim _{s \rightarrow \infty} s^{\frac{\sigma+1-p}{p}} s \frac{\int_{s}^{\infty} \frac{\int_{S_{0}}^{t} E(v) f(v) d v}{p(F(t))^{1+\frac{1}{p}}} d t+\int_{s}^{\infty} \frac{c d t}{p(F(t))^{1+\frac{1}{p}}}}{\int_{s}^{\infty} \frac{d t}{(F(t))^{\frac{1}{p}}}} \\
& =\frac{1}{p} \lim _{s \rightarrow \infty} \frac{s^{\frac{\sigma+1-p}{p}} \varsigma(s) \frac{\int_{S_{0}}^{s} E(v) f(v) d v}{s E(s) f(s)} \frac{s f(s)}{F(s)}+c \frac{s^{\frac{\sigma+1-p}{p}} \varsigma}{F(s)}}{1+\frac{\sigma+1-p}{p} \varsigma s^{-1}(F(s))^{\frac{1}{p}} \int_{s}^{\infty} \frac{d v}{(F(v))^{\frac{1}{p}}}}=\kappa_{2} .
\end{aligned}
$$

(iii) By a simple calculation, we get

$$
\begin{aligned}
& \lim _{s \rightarrow \infty} s^{\frac{\sigma+1-p}{p}} s\left(\frac{(F(s))^{1-\frac{1}{p}}}{f(s) \int_{s}^{\infty}(F(t))^{-\frac{1}{p}} d t}+\frac{p-\sigma-1}{p(\sigma+1)}\right) \\
& =\lim _{s \rightarrow \infty} s^{\frac{\sigma+1-p}{p}} s\left(\frac{F(s)}{s f(s)} \frac{s(F(s))^{-\frac{1}{p}}}{\int_{s}^{\infty}(F(t))^{-\frac{1}{p}} d t}+\frac{p-\sigma-1}{p(\sigma+1)}\right) \\
& =\lim _{s \rightarrow \infty} s^{\frac{\sigma+1-p}{p}} s\left(\left(\frac{F(s)}{s f(s)}-\frac{1}{\sigma+1}\right)\left(\frac{s(F(s))^{-\frac{1}{p}}}{\int_{s}^{\infty}(F(t))^{-\frac{1}{p}} d t}+\frac{p-\sigma-1}{p}\right)\right. \\
& \left.\quad-\frac{p-\sigma-1}{p}\left(\frac{F(s)}{s f(s)}-\frac{1}{\sigma+1}\right)+\frac{1}{\sigma+1}\left(\frac{s(F(s))^{-\frac{1}{p}}}{\int_{s}^{\infty}(F(t))^{-\frac{1}{p}} d t}+\frac{p-\sigma-1}{p}\right)\right) .
\end{aligned}
$$

Hence, by (i)-(ii), we get

$$
\lim _{s \rightarrow \infty} s^{\frac{\sigma+1-p}{p}} s\left(\frac{(F(s))^{1-\frac{1}{p}}}{f(s) \int_{s}^{\infty}(F(t))^{-\frac{1}{p}} d t}+\frac{p-\sigma-1}{p(\sigma+1)}\right)=\kappa_{3} .
$$

(iv) By (3.10), we see that

$$
\begin{aligned}
& \lim _{s \rightarrow \infty} s^{\frac{\sigma+1-p}{p}} s\left(\frac{f\left(A_{1} s\right)}{A_{1}^{p-1} f(s)}-A_{1}^{\sigma+1-p}\right) \\
& \quad=A_{1}^{\sigma+1-p} \lim _{s \rightarrow \infty} s^{\frac{\sigma+1-p}{p}} s(s) \int_{1}^{A_{1}} \frac{E(s u)}{E(s) u} d u .
\end{aligned}
$$

Hence, by (3.9), we reach

$$
\begin{aligned}
& \lim _{s \rightarrow \infty} s^{\frac{\sigma+1-p}{p}} \varsigma\left(\frac{f\left(A_{1} s\right)}{A_{1}^{p-1} f(s)}-A_{1}^{\sigma-p+1}\right) \\
& \quad=A_{1}^{p-1} \lim _{s \rightarrow \infty} s^{\frac{\sigma+1-p}{p}} s(s) \lim _{s \rightarrow \infty} \int_{1}^{A_{1}} \frac{E(s u)}{E(s) u} d u=\kappa_{4} .
\end{aligned}
$$

Lemma 3.5 Let $f$ satisfy $\left(f_{1}\right)-\left(f_{3}\right)$, and let $\left(f_{6}\right)$ hold, then

(i)

$$
\lim _{s \rightarrow \infty}(\ln s)^{\tau}\left(\frac{F(s)}{s f(s)}-\frac{1}{\sigma+1}\right)=\gamma_{1},
$$


where

$$
\gamma_{1}= \begin{cases}0 & \text { if } \eta<0 \\ -\frac{q_{3}}{(\sigma+1)(\sigma+1+\eta)} & \text { if } \eta=0\end{cases}
$$

(ii)

$$
\lim _{s \rightarrow \infty}(\ln s)^{\tau}\left(\frac{s(F(s))^{-\frac{1}{p}}}{\int_{s}^{\infty}(F(t))^{-\frac{1}{p}} d t}-\frac{\sigma+1-p}{p}\right)=\gamma_{2},
$$

where

$$
\gamma_{2}= \begin{cases}0 & \text { if } \eta<0 \\ \frac{q_{3}(\sigma+1)}{p(\eta+\sigma+1)} & \text { if } \eta=0\end{cases}
$$

(iii)

$$
\lim _{s \rightarrow \infty}(\ln s)^{\tau}\left(\frac{(F(s))^{1-\frac{1}{p}}}{f(s) \int_{s}^{\infty}(F(t))^{-\frac{1}{p}} d t}+\frac{p-\sigma-1}{p(\sigma+1)}\right)=\gamma_{3},
$$

where

$$
\gamma_{3}= \begin{cases}0 & \text { if } \eta<0 \\ \frac{q_{3}}{(\sigma+1)(\sigma+1+\eta)} & \text { if } \eta=0\end{cases}
$$

(iv)

$$
\lim _{s \rightarrow \infty}(\ln s)^{\tau}\left(\frac{f\left(A_{1} s\right)}{A_{1}^{p-1} f(s)}-A_{1}^{\sigma-p+1}\right)=\gamma_{4},
$$

where

$$
\gamma_{4}= \begin{cases}0 & \text { if } \eta<0 \\ q_{3} A_{1}^{\sigma-p+1} \ln A_{1} & \text { if } \eta=0\end{cases}
$$

Proof The proof is similar to the proof of Lemma 3.3, we omit it here.

Lemma 3.6 Iff satisfies $\left(f_{1}\right)-\left(f_{2}\right)$ and $\phi$ is the solution to problem (1.7), then

(i) $\phi^{\prime}(t)=-(q F(\phi(t)))^{\frac{1}{p}}, \phi(t)>0, t>0, \phi(0):=\lim _{t \rightarrow 0^{+}} \phi(t)=\infty$;

(ii) $\phi^{\prime \prime}(t)=p^{-1} q^{\frac{2}{p}} f(\phi(t))(F(\phi(t)))^{(2-p) / p}, t>0,\left|\phi^{\prime}(t)\right|^{p-2} \phi^{\prime \prime}(t)=\frac{q}{p} f(\phi(t))$;

(iii) $\phi \in \mathrm{NRVZ}_{-\frac{p}{\sigma+1-p}}$ and $\phi^{\prime} \in \mathrm{NRVZ}_{-\frac{\sigma+1}{\sigma+1-p}}$;

(iv) when $k \in \Lambda, \lim _{t \rightarrow 0^{+}} \frac{\ln t}{\ln (\phi(K(t)))}=-\frac{(\sigma+1-p) C_{k}}{p}$;

(v) when $k \in \Lambda$ with $C_{k} \in(0,1), \lim _{t \rightarrow 0^{+}} t(\phi(K(t)))^{\frac{\sigma+1-p}{p}}=\infty$ and $\lim _{t \rightarrow 0^{+}} \frac{(-\ln t)^{\beta}}{\phi(K(t))}=0$; furthermore, if $\varpi<\frac{p}{(\sigma+1-p) C_{k}}, \lim _{t \rightarrow 0^{+}} t^{\varpi} \phi(K(t))=\infty$;

(vi) when $k \in \Lambda_{3, \varsigma}, \lim _{t \rightarrow 0^{+}} \frac{1}{t^{\varsigma} \ln (\phi(K(t)))}=\frac{(\sigma+1-p) \varsigma D_{3 k}}{p(\varsigma+1)}$. 
Proof By the definition of $\phi$ and a direct calculation, we show that (i)-(ii) hold.

(iii) By (i)-(ii) and Lemma 3.2(iv)-(v), we have that

$$
\lim _{t \rightarrow 0^{+}} \frac{t \phi^{\prime \prime}(t)}{\phi^{\prime}(t)}=-\frac{1}{p} \lim _{s \rightarrow \infty} \frac{f(s) \int_{s}^{\infty} \frac{d v}{(F(v))^{\frac{1}{p}}}}{(F(s))^{1-\frac{1}{p}}}=-\frac{\sigma+1}{\sigma+1-p}
$$

and

$$
\lim _{t \rightarrow 0^{+}} \frac{t \phi^{\prime}(t)}{\phi(t)}=-\lim _{s \rightarrow \infty} \frac{(F(s))^{\frac{1}{p}}}{s} \int_{s}^{\infty} \frac{d v}{(F(v))^{\frac{1}{p}}}=-\frac{p}{\sigma+1-p},
$$

i.e., $\phi^{\prime} \in \mathrm{NRVZ}_{-\frac{p}{\sigma+1-p}}$ and $\phi \in \mathrm{NRVZ}_{-\frac{\sigma+1}{\sigma+1-p}}$ and (iii) follows.

(iv) By l'Hospital's rule, (iii), and Lemma 3.1(i), we see that

$$
\begin{aligned}
\lim _{t \rightarrow 0^{+}} \frac{\ln t}{\ln (\phi(K(t)))} & =\lim _{t \rightarrow 0^{+}} \frac{K(t)}{t k(t)} \frac{\phi(K(t))}{K(t) \phi^{\prime}(K(t))} \\
& =-\frac{\sigma+1-p}{p} C_{k} .
\end{aligned}
$$

(v) By Lemma 3.1(i), we see $K \in \mathrm{NRVZ}_{C_{k}^{-1}}$. It follows by Proposition 2.4 that $\phi^{\frac{\sigma+1-p}{p}} \circ$ $K \in \mathrm{NRVZ}_{-\frac{1}{C_{k}}}$ and $\phi \circ K \in \mathrm{NRVZ}_{-\frac{p}{(\sigma+1-p) C_{k}}}$. Since $C_{k} \in(0,1)$ and $\varpi<\frac{p}{(\sigma+1-p) C_{k}}$, the result follows by Proposition 2.3(ii).

(vi) By l'Hospital's rule and (iii), we obtain

$$
\begin{aligned}
\lim _{t \rightarrow 0^{+}} \frac{1}{t^{\varsigma} \ln (\phi(K(t)))} & =-\varsigma \lim _{t \rightarrow 0^{+}} \frac{K(t)}{t^{\varsigma+1} k(t)} \frac{\phi(K(t))}{K(t) \phi^{\prime}(K(t))} \\
& =\frac{(\sigma+1-p) \varsigma}{p} \lim _{t \rightarrow 0^{+}} \frac{K(t)}{t^{\varsigma+1} k(t)}=\frac{(\sigma+1-p) \varsigma D_{3 k}}{p(\varsigma+1)} .
\end{aligned}
$$

Lemma 3.7 Under the hypotheses in Theorem 1.1, let $\phi$ be the solution to problem (1.7).

(i)

$$
\lim _{t \rightarrow 0^{+}}(-\ln t)^{\beta}\left(\frac{\phi^{\prime}(K(t)) k^{\prime}(t)}{\phi^{\prime \prime}\left(K(t) k^{2}(t)\right.}+\frac{(\sigma+1-p)\left(1-C_{k}\right)}{\sigma+1}\right)=\chi_{1},
$$

where

$$
\chi_{1}= \begin{cases}\frac{(\sigma+1-p) D_{1 k}}{\sigma+1} & \text { if (i) or (ii) holds } \\ \frac{-q_{1} \xi_{1} p\left(1-C_{k}\right)}{(\sigma+1)(\sigma+1+\eta)}+\frac{(\sigma+1-p) D_{1 k}}{\sigma+1} & \text { if (iii) holds }\end{cases}
$$

(ii)

$$
\lim _{t \rightarrow 0^{+}}(-\ln t)^{\beta}\left(\frac{f\left(A_{1} \phi(K(t))\right)}{A_{1}^{p-1} f(\phi(K(t)))}-A_{1}^{\sigma-p+1}\right)=\chi_{2},
$$


where

$$
\chi_{2}= \begin{cases}0 & \text { if (i) or (ii) holds, } \\ \xi_{1} q_{1} A_{1}^{\sigma-p+1} \ln A_{1} & \text { if (iii) holds; }\end{cases}
$$

where $\xi_{1}=p^{-\beta}\left((p-1-\sigma) C_{k}\right)^{\beta}$.

Proof (i) By the definition of $\phi$, Lemma 3.3(iii), and Lemma 3.7(iv), we arrive at

$$
\begin{aligned}
\lim _{t \rightarrow 0^{+}} & (-\ln t)^{\beta}\left(\frac{\phi^{\prime}(K(t)) k^{\prime}(t)}{\phi^{\prime \prime}(K(t)) k^{2}(t)}+\frac{(\sigma+1-p)\left(1-C_{k}\right)}{\sigma+1}\right) \\
= & \lim _{t \rightarrow 0^{+}}(-\ln t)^{\beta}\left(\frac{K(t) k^{\prime}(t)}{k^{2}(t)}-\left(1-C_{k}\right)\right)\left(\frac{\phi^{\prime}(K(t))}{\phi^{\prime \prime}(K(t)) K(t)}+\frac{\sigma+1-p}{\sigma+1}\right) \\
& +\left(1-C_{k}\right) \lim _{t \rightarrow 0^{+}}(-\ln t)^{\beta}\left(\frac{\phi^{\prime}(K(t))}{\phi^{\prime \prime}(K(t)) K(t)}+\frac{\sigma+1-p}{\sigma+1}\right) \\
& -\frac{\sigma+1-p}{\sigma+1} \lim _{t \rightarrow 0^{+}}(-\ln t)^{\beta}\left(\frac{K(t) k^{\prime}(t)}{k^{2}(t)}-\left(1-C_{k}\right)\right) \\
= & \left(1-C_{k}\right) \lim _{t \rightarrow 0^{+}}(-\ln \phi(K(t)))^{\beta}\left(\frac{-p(F(\phi(K(t))))^{1-\frac{1}{p}}}{f(\phi(K(t))) \int_{\phi(K(t))}^{\infty}(F(v))^{-\frac{1}{p}} d v}+\frac{\sigma+1-p}{\sigma+1}\right) \\
& \times\left(\frac{-\ln t}{\ln \phi(K(t))}\right)^{\beta}-\frac{\sigma+1-p}{\sigma+1} \lim _{t \rightarrow 0^{+}}(-\ln t)^{\beta}\left(\frac{K(t) k^{\prime}(t)}{k^{2}(t)}-\left(1-C_{k}\right)\right) \\
= & \chi_{1} .
\end{aligned}
$$

(ii) By Lemma 3.3(iv) and Lemma 3.7(iv), we infer that

$$
\begin{aligned}
& \lim _{t \rightarrow 0^{+}}(-\ln t)^{\beta}\left(\frac{f\left(A_{1} \phi(K(t))\right)}{A_{1}^{p-1} f(\phi(K(t)))}-A_{1}^{\sigma-p+1}\right) \\
& \quad=\lim _{t \rightarrow 0^{+}}(\ln (\phi(K(t))))^{\beta}\left(\frac{f\left(A_{1} \phi(K(t))\right)}{A_{1}^{p-1} f(\phi(K(t)))}-A_{1}^{\sigma-p+1}\right)\left(\frac{-\ln t}{\ln \phi(K(t))}\right)^{\beta} \\
& \quad=\chi_{2} .
\end{aligned}
$$

Lemma 3.8 Under the hypotheses in Theorem 1.2, let $\phi$ be the solution to problem (1.7).

Then

(i)

$$
\lim _{t \rightarrow 0^{+}} t^{-\varpi}\left(\frac{\phi^{\prime}(K(t)) k^{\prime}(t)}{\phi^{\prime \prime}(K(t)) k^{2}(t)}+\frac{(\sigma+1-p)\left(1-C_{k}\right)}{\sigma+1}\right)=\frac{\sigma+1-p}{\sigma+1} D_{2 k} \text { Heaviside }(\theta-\varsigma) ;
$$

(ii)

$$
\lim _{t \rightarrow 0^{+}} t^{-\varpi}\left(\frac{f\left(A_{1} \phi(K(t))\right)}{A_{1}^{p-1} f(\phi(K(t)))}-A_{1}^{\sigma-p+1}\right)=0 .
$$

Proof (i) By the definition of $\phi$, Lemma 3.4(iii), and Lemma 3.7(v), we arrive at

$$
\lim _{t \rightarrow 0^{+}} t^{-\varpi}\left(\frac{\phi^{\prime}(K(t)) k^{\prime}(t)}{\phi^{\prime \prime}(K(t)) k^{2}(t)}+\frac{(\sigma+1-p)\left(1-C_{k}\right)}{\sigma+1}\right)
$$




$$
\begin{aligned}
= & \lim _{t \rightarrow 0^{+}} t^{-\varpi}\left(\frac{K(t) k^{\prime}(t)}{k^{2}(t)}-\left(1-C_{k}\right)\right)\left(\frac{\phi^{\prime}(K(t))}{\phi^{\prime \prime}(K(t)) K(t)}+\frac{\sigma+1-p}{\sigma+1}\right) \\
& +\left(1-C_{k}\right) \lim _{t \rightarrow 0^{+}} t^{-\varpi}\left(\frac{\phi^{\prime}(K(t))}{\phi^{\prime \prime}(K(t)) K(t)}+\frac{\sigma+1-p}{\sigma+1}\right) \\
& -\frac{\sigma+1-p}{\sigma+1} \lim _{t \rightarrow 0^{+}} t^{-\varpi}\left(\frac{K(t) k^{\prime}(t)}{k^{2}(t)}-\left(1-C_{k}\right)\right) \\
= & \left(1-C_{k}\right) \lim _{t \rightarrow 0^{+}}(\phi(K(t)))^{\frac{(\sigma+1-p)}{p}}\left(\frac{-p(F(\phi(K(t))))^{1-\frac{1}{p}}}{f(\phi(K(t))) \int_{\phi(K(t))}^{\infty}(F(v))^{-\frac{1}{p}} d v}+\frac{\sigma+1-p}{\sigma+1}\right) \\
& \times\left((\phi(K(t)))^{\frac{\sigma+1-p}{p}} t\right)^{-\varpi}-\frac{\sigma+1-p}{\sigma+1} \lim _{t \rightarrow 0^{+}} t^{-\varpi}\left(\frac{K(t) k^{\prime}(t)}{k^{2}(t)}-\left(1-C_{k}\right)\right) \\
= & \frac{\sigma+1-p}{\sigma+1} D_{2 k} \operatorname{Heaviside}(\theta-\varsigma) .
\end{aligned}
$$

(ii) By Lemma 3.4(iv) and Lemma 3.7(v), we infer that

$$
\begin{aligned}
& \lim _{t \rightarrow 0^{+}} t^{-\varpi}\left(\frac{f\left(A_{1} \phi(K(t))\right)}{A_{1}^{p-1} f(\phi(K(t)))}-A_{1}^{\sigma-p+1}\right) \\
& \quad=\lim _{t \rightarrow 0^{+}}(\phi(K(t)))^{\frac{(\sigma+1-p)}{p} \varpi}\left(\frac{f\left(A_{1} \phi(K(t))\right)}{A_{1}^{p-1} f(\phi(K(t)))}-A_{1}^{\sigma-p+1}\right)\left((\phi(K(t)))^{\frac{\sigma+1-p}{p}} t\right)^{-\varpi} \\
& \quad=0 .
\end{aligned}
$$

Lemma 3.9 Under the hypotheses in Theorem 1.3. Let $\phi$ be the solution to problem (1.7).

Then

(i)

$$
\lim _{t \rightarrow 0^{+}} t^{-\varpi}\left(\frac{\phi^{\prime}(K(t)) k^{\prime}(t)}{\phi^{\prime \prime}(K(t)) k^{2}(t)}+\frac{\sigma+1-p}{\sigma+1}\right)=\chi_{3},
$$

where

$$
\chi_{3}= \begin{cases}\frac{\sigma+1-p}{\sigma+1} D_{3 k} \text { Heaviside }(\theta-\varsigma) & \text { if (i) or (ii) holds } \\ -\frac{\xi_{2} q_{3} p}{(\sigma+1)(\sigma+1+\eta)}+\frac{(\sigma+1-p) D_{3 k}}{\sigma+1} \operatorname{Heaviside}(\theta-\varsigma), & \text { if (iii) holds }\end{cases}
$$

(ii)

$$
\lim _{t \rightarrow 0^{+}} t^{-\varpi}\left(\frac{f\left(A_{1} \phi(K(t))\right)}{A_{1}^{p-1} f(\phi(K(t)))}-A_{1}^{\sigma-p+1}\right)=\chi_{4},
$$

where

$$
\chi_{4}= \begin{cases}0 & \text { if (i) or (ii) holds, } \\ \xi_{2} q_{3} A_{1}^{\sigma-p+1} \ln A_{1} & \text { if (iii) holds; }\end{cases}
$$

where $\xi_{2}=(p(\varsigma+1))^{-\tau}\left((\sigma+1-p) \varsigma D_{3 k}\right)^{\tau}$. 
Proof (i) By the definition of $\phi$, Lemma 3.5(iii), and Lemma 3.7(vi), we arrive at

$$
\begin{aligned}
\lim _{t \rightarrow 0^{+}} & t^{-\varpi}\left(\frac{\phi^{\prime}(K(t)) k^{\prime}(t)}{\phi^{\prime \prime}(K(t)) k^{2}(t)}+\frac{\sigma+1-p}{\sigma+1}\right) \\
= & \lim _{t \rightarrow 0^{+}} t^{-\varpi}\left(\frac{K(t) k^{\prime}(t)}{k^{2}(t)}-1\right)\left(\frac{\phi^{\prime}(K(t))}{\phi^{\prime \prime}(K(t)) K(t)}+\frac{\sigma+1-p}{\sigma+1}\right) \\
& +\lim _{t \rightarrow 0^{+}} t^{-\varpi}\left(\frac{\phi^{\prime}(K(t))}{\phi^{\prime \prime}(K(t)) K(t)}+\frac{\sigma+1-p}{\sigma+1}\right) \\
& -\frac{\sigma+1-p}{\sigma+1} \lim _{t \rightarrow 0^{+}} t^{-\varpi}\left(\frac{K(t) k^{\prime}(t)}{k^{2}(t)}-1\right) \\
= & \lim _{t \rightarrow 0^{+}}(\ln (\phi(K(t))))^{\tau}\left(\frac{-p(F(\phi(K(t))))^{1-\frac{1}{p}}}{f(\phi(K(t))) \int_{\phi(K(t))}^{\infty}(F(v))^{-\frac{1}{p}} d v}+\frac{\sigma+1-p}{\sigma+1}\right) \\
& \quad \times\left(\ln (\phi(K(t))) t^{5}\right)^{-\tau}-\frac{\sigma+1-p}{\sigma+1} \lim _{t \rightarrow 0^{+}} t^{-\varpi}\left(\frac{K(t) k^{\prime}(t)}{k^{2}(t)}-1\right) \\
= & \chi_{3} .
\end{aligned}
$$

(ii) By Lemma 3.5(iv) and Lemma 3.7(vi), we infer that

$$
\begin{aligned}
& \lim _{t \rightarrow 0^{+}} t^{-\varpi}\left(\frac{f\left(A_{1} \phi(K(t))\right)}{A_{1}^{p-1} f(\phi(K(t)))}-A_{1}^{\sigma-p+1}\right) \\
& \quad=\lim _{t \rightarrow 0^{+}}(\ln (\phi(K(t))))^{\tau}\left(\frac{f\left(A_{1} \phi(K(t))\right)}{A_{1}^{p-1} f(\phi(K(t)))}-A_{1}^{\sigma-p+1}\right)\left(\ln (\phi(K(t))) t^{\varsigma}\right)^{-\tau} \\
& \quad=\chi_{4} .
\end{aligned}
$$

\section{Proof of Theorem 1.1}

First, we need the following comparison principle for weak solutions to quasilinear equations (see [33] for a proof).

Lemma 4.1 (Weak comparison principle) Let $D \subset \mathbb{R}^{N}$ be a bounded domain, $G: D \times \mathbb{R} \rightarrow$ $\mathbb{R}$ be non-increasing in the second variable and continuous. Let $u, w \in W^{1, p}(D)$ satisfy the respective inequalities

$$
\begin{aligned}
& \int_{D}|\nabla u|^{p-2} \nabla u \cdot \nabla \phi \leq \int_{D} G(x, u) \phi \quad \text { and } \\
& \int_{D}|\nabla w|^{p-2} \nabla w \cdot \nabla \phi \geq \int_{D} G(x, w) \phi
\end{aligned}
$$

for all non-negative $\phi \in W_{0}^{1, p}(D)$. Then the inequality $u \leq w$ on $\partial D$ implies $u \leq w$ in $D$.

Next fix $\varepsilon>0$. For any $\delta>0$, we define $\Omega_{\delta}=\{x \in \Omega: 0<d(x)<\delta\}$. Since $\Omega$ is $C^{2}$-smooth, choose $\delta_{1} \in\left(0, \delta_{0}\right)$ such that $d \in C^{2}\left(\Omega_{\delta_{1}}\right)$ and

$$
|\nabla d(x)|=1, \quad \Delta d(x)=-(N-1) H(\bar{x})+o(1), \quad \forall x \in \Omega_{\delta_{1}}
$$

where, for all $x \in \Omega_{\delta_{1}}, \bar{x}$ denotes the unique point of the boundary such that $d(x)=|x-\bar{x}|$ and $H(\bar{x})$ denotes the mean curvature of the boundary at that point. 
Let $0<a_{0}<1$ and

$$
w_{ \pm}=A_{1} \phi(K(d(x)))\left(1+\left(A_{2} \pm \varepsilon\right)(-\ln (d(x)))^{-\beta}\right), \quad x \in \Omega_{\delta_{1}} .
$$

By the Lagrange mean value theorem, we obtain that there exist $\lambda_{ \pm} \in(0,1)$ and

$$
\Phi_{ \pm}(d(x))=A_{1} \phi(K(d(x)))\left(1+\lambda_{ \pm}\left(A_{2} \pm \varepsilon\right)(-\ln (d(x)))^{-\beta}\right)
$$

such that, for $x \in \Omega_{\delta_{1}}$,

$$
f\left(w_{ \pm}(x)\right)=f\left(A_{1} \phi(K(d(x)))\right)+A_{1}\left(A_{2} \pm \varepsilon\right) \phi(K(d(x))) f^{\prime}\left(\Phi_{ \pm}(d(x))\right)(-\ln (d(x)))^{-\beta} .
$$

Since $f \in \mathrm{NRV}_{\sigma}$, by Proposition 2.1 we obtain

$$
\lim _{d(x) \rightarrow 0} \frac{f\left(A_{1} \phi(K(d(x)))\right)}{f\left(\Phi_{ \pm}(d(x))\right)}=\lim _{d(x) \rightarrow 0} \frac{f^{\prime}\left(A_{1} \phi(K(d(x)))\right)}{f^{\prime}\left(\Phi_{ \pm}(d(x))\right)}=1 .
$$

Define $r=d(x)$ and

$$
\begin{aligned}
I_{1}(r)= & (-\ln r)^{\beta}(p-1)\left(1+\frac{\phi^{\prime}(K(r)) k^{\prime}(r)}{\phi^{\prime \prime}(K(r)) k^{2}(r)}-\frac{f\left(A_{1} \phi(K(r))\right)}{A_{1}^{p-1} f(\phi(K(r)))}\right) ; \\
I_{2 \pm}(r)= & \left(A_{2} \pm \varepsilon\right)(p-1)\left(1+\frac{\phi^{\prime}(K(r)) k^{\prime}(r)}{\phi^{\prime \prime}(K(r)) k^{2}(r)}+(p-2) \frac{\phi^{\prime}(K(r)) k^{\prime}(r)}{\phi^{\prime \prime}(K(r)) k^{2}(r)}\right. \\
& \left.+(p-2)-A_{1} \frac{f^{\prime}\left(\Phi_{ \pm}(r)\right)}{f^{\prime}\left(A_{1} \phi(K(r))\right)} \frac{\phi(K(r)) f^{\prime}\left(A_{1} \phi(K(r))\right)}{A_{1}^{p-1} f(\phi(K(r)))}\right) ; \\
I_{3 \pm}(x)= & \beta\left(A_{2} \pm \varepsilon\right) \frac{\phi(K(r))}{\phi^{\prime \prime}(K(r)) k^{2}(r) r^{2}}\left((p-1)(\beta+1)(-\ln r)^{-2}+(-\ln r)^{-1} \Delta d(x)\right. \\
& \left.-(p-1)(-\ln r)^{-1} r^{-2}\right)-(p-1)\left(c \mp a_{0} \varepsilon\right) \frac{f\left(A_{1} \phi(K(r))\right)}{A_{1}^{\sigma-p+1} f(\phi(K(r)))}(-\ln r)^{\beta} r^{\theta} \\
& +2 \frac{\phi^{\prime}(K(r))}{\phi^{\prime \prime}(K(r)) k(r)}\left(\left(A_{2} \pm \varepsilon\right)\left(\Delta d(x)+2 \beta(-\ln r)^{-1} r^{-1}\right)+\Delta d(x)(-\ln r)^{\beta}\right) \\
& -A_{1}(p-1)\left(A_{2} \pm \varepsilon\right)\left(c \mp a_{0} \varepsilon\right) r^{\theta} \frac{f^{\prime}\left(\Phi_{ \pm}(r)\right)}{f^{\prime}\left(A_{1} \phi(K(r))\right)} \frac{\phi(K(r)) f^{\prime}\left(A_{1} \phi(K(r))\right)}{A_{1}^{\sigma-p+1} f(\phi(K(r)))} \\
& +(p-2)\left(A_{2}+\varepsilon\right)^{2}\left(1+\frac{\phi^{\prime}(K(r)) k^{\prime}(r)}{\phi^{\prime \prime}(K(r)) k^{2}(r)}\right)(-\ln r)^{\beta}+o\left((-\ln r)^{\beta}\right) .
\end{aligned}
$$

By Lemmas 3.1, 3.6, and 3.7, combining with the choices of $A_{1}, A_{2}, \xi_{1}$ in Theorem 1.1, we see the following.

Lemma 4.2 Under the hypotheses in Theorem 1.1,

(i) $\lim _{r \rightarrow 0} I_{1}(r)=\lambda_{1}$, where

$$
\lambda_{1}= \begin{cases}\frac{(p-1)(\sigma+1-p)}{\sigma+1} D_{1 k}:=\Theta_{1} & \text { if (i) or (ii) holds } \\ \Theta_{1}-(p-1) q_{1} \xi_{1}\left(\frac{p\left(1-C_{k}\right)}{(\sigma+1)(\sigma+1+\eta)}+A_{1}^{\sigma-p+1} \ln A_{1}\right) & \text { if (iii) holds }\end{cases}
$$

(ii) $\lim _{r \rightarrow 0} I_{2 \pm}(r)=\left(A_{2} \pm \varepsilon\right)(p-1)(p-1-\sigma) \frac{p+(\sigma+1-p) C_{k}}{\sigma+1}$; 
(iii) $\lim _{d(x) \rightarrow 0} I_{3 \pm}(x)=0$;

(iv) $\lim _{d(x) \rightarrow 0}\left(I_{1}(r)+I_{2 \pm}(r)+I_{3 \pm}(x)\right)= \pm \varepsilon(p-1)(p-1-\sigma) \frac{p+(\sigma+1-p) C_{k}}{\sigma+1}$.

Proof of Theorem 1.1 By $\left(b_{1}\right),\left(b_{2}\right)$, Lemma 4.2, and $K \in C\left[0, \delta_{0}\right)$ with $K(0)=0$, we see that there are $\delta_{1 \varepsilon}, \delta_{2 \varepsilon} \in\left(0, \min \left\{1, \delta_{1} / 2\right\}\right)$ (which corresponds to $\varepsilon$ ) sufficiently small such that

(1) $0 \leq K(r) \leq 2 \delta_{1 \varepsilon}, r \in\left(0,2 \delta_{2 \varepsilon}\right)$;

(2) $k^{p}(d(x))\left(1+\left(c-a_{0} \varepsilon\right)(d(x))^{\theta}\right) \leq b(x) \leq k^{p}(d(x))\left(1+\left(c+a_{0} \varepsilon\right)(d(x))^{\theta}\right), x \in \Omega_{2 \delta_{1 \varepsilon}}$;

(3) $I_{1}(r)+I_{2+}(r)+I_{3+}(x) \leq 0, \forall(x, r) \in \Omega_{2 \delta_{1 \varepsilon}} \times\left(0,2 \delta_{2 \varepsilon}\right)$;

(4) $I_{1}(r)+I_{2-}(r)+I_{3-}(x) \geq 0, \forall(x, r) \in \Omega_{2 \delta_{1 \varepsilon}} \times\left(0,2 \delta_{2 \varepsilon}\right)$.

Now we define

$$
\begin{aligned}
& \left\{\begin{array}{l}
\sigma \in\left(0, \delta_{1 \varepsilon}\right), \quad D_{\sigma}^{-}=\Omega_{2 \delta_{1 \varepsilon}} / \bar{\Omega}_{\sigma}, \quad D_{\sigma}^{+}=\Omega_{2 \delta_{1 \varepsilon}-\sigma}, \\
d_{1}(x)=d(x)-\sigma, \quad d_{2}(x)=d(x)+\sigma ;
\end{array}\right. \\
& \bar{u}_{\varepsilon}=A_{1} \phi\left(K\left(d_{1}(x)\right)\right)\left(1+\left(A_{2}+\varepsilon\right)\left(-\ln \left(d_{1}(x)\right)\right)^{-\beta}\right), \quad x \in D_{\sigma}^{-} .
\end{aligned}
$$

Then, for $x \in D_{\sigma}^{-}$,

$$
f\left(\bar{u}_{\varepsilon}(x)\right)=f\left(A_{1} \phi\left(K\left(d_{1}(x)\right)\right)\right)+A_{1}\left(A_{2}+\varepsilon\right) \phi\left(K\left(d_{1}(x)\right)\right) f^{\prime}\left(\Phi_{+}\left(d_{1}(x)\right)\right)\left(-\ln \left(d_{1}(x)\right)\right)^{-\beta},
$$

where $\lambda_{+} \in(0,1)$ and

$$
\Phi_{+}\left(d_{1}(x)\right)=A_{1} \phi\left(K\left(d_{1}(x)\right)\right)\left(1+\lambda_{+}\left(A_{2}+\varepsilon\right)\left(-\left(\ln \left(d_{1}(x)\right)\right)\right)^{-\beta}\right) .
$$

Before we prove the theorem, let us make note of the following. Suppose that $z$ is a $C^{2}$ function on a domain $\Omega$ in $\mathbb{R}^{N}$, and $v=\phi(z)$, where $\phi$ is uniquely determined by (1.7). Direct computation shows that

$$
\Delta_{p} v=(p-1)\left|\phi^{\prime}(z)\right|^{p-2} \phi^{\prime \prime}(z)|\nabla z|^{p}+\left|\phi^{\prime}(z)\right|^{p-2} \phi^{\prime}(z) \triangle_{p} z
$$

Then, combining with (4.4), it follows that, for $x \in D_{\sigma}^{-}$,

$$
\begin{aligned}
& \Delta_{p} \bar{u}_{\varepsilon}(x)-k^{p}\left(d_{1}(x)\right)\left(1+\left(c-a_{0} \varepsilon\right)\left(d_{1}(x)\right)^{\theta}\right) f\left(\bar{u}_{\varepsilon}(x)\right) \\
& \quad=A_{1}^{p-1}\left|\phi^{\prime}(K(r))\right|^{p-2} \phi^{\prime \prime}(K(r)) k^{p}(r)(-\ln r)^{-\beta}\left(I_{1}(r)+I_{2+}(r)+I_{3+}(x)\right) \\
& \quad \leq 0
\end{aligned}
$$

where $r=d_{1}(x)$, i.e., $\bar{u}_{\varepsilon}$ is a supersolution of equation (1.1) in $D_{\sigma}^{-}$.

In a similar way, we can show that

$$
\underline{u}_{\varepsilon}=A_{1} \phi\left(K\left(d_{2}(x)\right)\right)\left(1+\left(A_{2}-\varepsilon\right)\left(-\ln \left(d_{2}(x)\right)\right)^{-\beta}\right), \quad x \in D_{\sigma}^{+},
$$

is a subsolution of equation (1.1) in $D_{\sigma}^{+}$.

Now let $u$ be an arbitrary solution of problem (1.1), and let $M>0$ be sufficiently large such that

$$
u(x) \leq M+\quad \text { on } d(x)=2 \delta_{1 \varepsilon} \quad \text { and } \quad \underline{u}_{\varepsilon}(x) \leq u(x)+M \quad \text { on } d(x)=2 \delta_{1 \varepsilon}-\sigma .
$$


We observe that $\bar{u}_{\varepsilon}(x) \rightarrow \infty$ as $d_{1}(x) \rightarrow \sigma$, and $\left.u\right|_{\partial \Omega}=+\infty>\left.u_{\varepsilon}\right|_{\partial \Omega}$. It follows from Lemma 4.1 (the weak comparison principle) that

$$
u \leq M+\bar{u}_{\varepsilon} \quad \text { in } D_{\sigma}^{-} \quad \text { and } \quad \underline{u}_{\varepsilon} \leq M+u \quad \text { in } D_{\sigma}^{+} .
$$

Hence, by letting $\sigma \rightarrow 0$, we have, for $x \in \Omega_{2 \delta_{1 \varepsilon}}$,

$$
A_{2}-\varepsilon-\frac{M(-\ln (d(x)))^{\beta}}{A_{1} \phi(K(d(x)))} \leq(-\ln (d(x)))^{\beta}\left(\frac{u(x)}{A_{1} \phi(K(d(x)))}-1\right)
$$

and

$$
(-\ln (d(x)))^{\beta}\left(\frac{u(x)}{A_{1} \phi(K(d(x)))}-1\right) \leq A_{2}+\varepsilon+\frac{M(-\ln (d(x)))^{\beta}}{A_{1} \phi(K(d(x)))} .
$$

Consequently, by Lemma 3.6(vi)

$$
\begin{aligned}
& A_{2}-\varepsilon \leq \lim _{d(x) \rightarrow 0} \inf (-\ln (d(x)))^{\beta}\left(\frac{u(x)}{A_{1} \phi(K(d(x)))}-1\right) \\
& \lim _{d(x) \rightarrow 0} \sup (-\ln (d(x)))^{\beta}\left(\frac{u(x)}{A_{1} \phi(K(d(x)))}-1\right) \leq A_{2}+\varepsilon .
\end{aligned}
$$

Thus letting $\varepsilon \rightarrow 0$, we obtain (1.8). The proof is finished.

\section{Proof of Theorem 1.2}

In this section, we prove Theorem 1.2.

Let $0<a_{0}<1$ and

$$
w_{ \pm}=A_{1} \phi(K(d(x)))\left(1+\left(A_{3} \pm \varepsilon\right)(d(x))^{\sigma}\right), \quad x \in \Omega_{\delta_{1}} .
$$

By the Lagrange mean value theorem, we obtain that there exist $\lambda_{ \pm} \in(0,1)$ and

$$
\Phi_{ \pm}(d(x))=A_{1} \phi(K(d(x)))\left(1+\lambda_{ \pm}\left(A_{3} \pm \varepsilon\right)(d(x))^{\varpi}\right)
$$

such that, for $x \in \Omega_{\delta_{1}}$,

$$
f\left(w_{ \pm}(x)\right)=f\left(A_{1} \phi(K(d(x)))\right)+A_{1}\left(A_{3} \pm \varepsilon\right) \phi(K(d(x))) f^{\prime}\left(\Phi_{ \pm}(d(x))\right)(d(x))^{\varpi} .
$$

Since $f \in \mathrm{NRV}_{\sigma}$, by Proposition 2.1 we obtain

$$
\lim _{d(x) \rightarrow 0} \frac{f\left(A_{1} \phi(K(d(x)))\right)}{f\left(\Phi_{ \pm}(d(x))\right)}=\lim _{d(x) \rightarrow 0} \frac{f^{\prime}\left(A_{1} \phi(K(d(x)))\right)}{f^{\prime}\left(\Phi_{ \pm}(d(x))\right)}=1 .
$$

Define $r=d(x)$ and

$$
\begin{aligned}
& I_{1}(r)=r^{-\varpi}(p-1)\left(1+\frac{\phi^{\prime}(K(r)) k^{\prime}(r)}{\phi^{\prime \prime}(K(r)) k^{2}(r)}-\frac{f\left(A_{1} \phi(K(r))\right)}{A_{1}^{p-1} f(\phi(K(r)))}\right) ; \\
& I_{2 \pm}(r)=\left(A_{3} \pm \varepsilon\right)(p-1)\left(p-1+\frac{\phi^{\prime}(K(r)) k^{\prime}(r)}{\phi^{\prime \prime}(K(r)) k^{2}(r)}+2 \varpi \frac{\phi^{\prime}(K(r))}{\phi^{\prime \prime}(K(r)) k(r) r}\right.
\end{aligned}
$$




$$
\begin{aligned}
& +\frac{\varpi(\varpi-1) \phi(K(r))}{\phi^{\prime \prime}(K(r)) k^{2}(r) r^{2}} \\
& -A_{1} \frac{f^{\prime}\left(\Phi_{ \pm}(r)\right)}{f^{\prime}\left(A_{1} \phi(K(r))\right)} \frac{\phi(K(r)) f^{\prime}\left(A_{1} \phi(K(r))\right)}{A_{1}^{p-1} f(\phi(K(r)))} \\
& +(p-2)\left(\frac{\varpi \phi(K(r))}{\phi^{\prime}(K(r)) k(r) r}+\frac{\phi^{\prime}(K(r)) k^{\prime}(r)}{\phi^{\prime \prime}(K(r)) k^{2}(r)}\right. \\
& \left.\left.+\varpi \frac{\phi(K(r))}{\phi^{\prime}(K(r)) k(r)} \frac{\phi^{\prime}(K(r)) k^{\prime}(r)}{\phi^{\prime \prime}(K(r)) k^{2}(r) r}\right)+o\left(s^{\varpi}\right)\right) ; \\
I_{3 \pm}(x)= & \Delta d(x)\left(\frac{\phi^{\prime}(K(r))}{\phi^{\prime \prime}(K(r)) k(r)}\left(r^{-\varpi}+\left(A_{3} \pm \varepsilon\right)\right)+\varpi r^{-1}\left(A_{3} \pm \varepsilon\right) \frac{\phi(K(r))}{\phi^{\prime \prime}(K(r)) k^{2}(r)}\right) ; \\
I_{4 \pm}(r)= & \left(c \mp a_{0} \varepsilon\right)(p-1) r^{\theta}\left(\frac{f\left(A_{1} \phi(K(r))\right)}{A_{1}^{p-1} f(\phi(K(r)))} r^{-\varpi}-\left(A_{3} \pm \varepsilon\right) \frac{f^{\prime}\left(\Phi_{ \pm}(r)\right) \phi(K(r))}{A_{1}^{p-1} f(\phi(K(r)))}\right) .
\end{aligned}
$$

By Lemmas 3.1,3.6, and 3.8, combining with the choices of $A_{1}, A_{3}$ in Theorem 1.2, we see the following.

Lemma 5.1 Under the hypotheses in Theorem 1.2,

(i) $\lim _{r \rightarrow 0} I_{1}(r)=\frac{(p-1)(\sigma+1-p)}{\sigma+1} D_{2 k}$ Heaviside $(\theta-\varsigma)$;

(ii) $\lim _{r \rightarrow 0} I_{2 \pm}(r)=\left(A_{3} \pm \varepsilon\right)(p-1) \chi$;

(iii) $\lim _{d(x) \rightarrow 0} I_{3 \pm}(x)=0$;

(iv) $\lim _{r \rightarrow 0} I_{4 \pm}(r)=\left(c \mp a_{0} \varepsilon\right)(p-1) \frac{C_{k}(\sigma+1-p)+p}{\sigma+1} \operatorname{Heaviside}(\varsigma-\theta)$;

(v) $\lim _{d(x) \rightarrow 0}\left(I_{1}(r)+I_{2 \pm}(r)+I_{3 \pm}(x)+I_{4 \pm}(r)\right)=$ $\pm \varepsilon(p-1)\left(\chi-a_{0} \frac{C_{k}(\sigma+1-p)+p}{\sigma+1}\right.$ Heaviside $\left.(\varsigma-\theta)\right)$,

where

$$
\chi=\frac{\sigma+1-p}{p(\sigma+1)}\left(C_{k}(\sigma+1-p)\left(\varpi(\varpi+1) C_{k}-p\left(1+\varpi C_{k}\right)\right)-p^{2}\left(1+\varpi C_{k}\right)\right) .
$$

Proof of Theorem 1.2 Using a proof similar to that for Theorem 1.1, let

$$
\bar{u}_{\varepsilon}=A_{1} \phi\left(K\left(d_{1}(x)\right)\right)\left(1+\left(A_{3}+\varepsilon\right)\left(d_{1}(x)\right)^{\varpi}\right), \quad x \in D_{\sigma}^{-} .
$$

Then, by a direct calculation, we have, for $x \in D_{\sigma}^{-}$,

$$
\begin{aligned}
& \Delta \bar{u}_{\varepsilon}(x)-k^{p}\left(d_{1}(x)\right)\left(1+\left(c-a_{0} \varepsilon\right)\left(d_{1}(x)\right)^{\theta}\right) f\left(\bar{u}_{\varepsilon}(x)\right) \\
& \quad=A_{1}^{p-1}\left|\phi^{\prime}(K(r))\right|^{p-2} \phi^{\prime \prime}(K(r)) k^{p}(r) r^{\varpi}\left(I_{1}(r)+I_{2+}(r)+I_{3+}(x)+I_{4+}(r)\right) \\
& \quad \leq 0
\end{aligned}
$$

where $r=d_{1}(x)$, i.e., $\bar{u}_{\varepsilon}$ is a supersolution of equation (1.1) in $D_{\sigma}^{-}$.

In a similar way, we can show that

$$
\underline{u}_{\varepsilon}=A_{1} \phi\left(K\left(d_{2}(x)\right)\right)\left(1+\left(A_{3}-\varepsilon\right)\left(d_{2}(x)\right)^{\varpi}\right), \quad x \in D_{\sigma}^{+},
$$

is a subsolution of equation (1.1) in $D_{\sigma}^{+}$.

Using a proof similar to that for Theorem 1.1, we can obtain, for $x \in \Omega_{2 \delta_{1 \varepsilon}}$,

$$
A_{3}-\varepsilon-\frac{M(d(x))^{-\sigma}}{A_{1} \phi(K(d(x)))} \leq(d(x))^{-\sigma}\left(\frac{u(x)}{A_{1} \phi(K(d(x)))}-1\right)
$$


and

$$
(d(x))^{-\varpi}\left(\frac{u(x)}{A_{1} \phi(K(d(x)))}-1\right) \leq A_{3}+\varepsilon+\frac{M(d(x))^{-\varpi}}{A_{1} \phi(K(d(x)))}
$$

Consequently, by Lemma 3.6,

$$
\begin{gathered}
A_{3}-\varepsilon \leq \lim _{d(x) \rightarrow 0} \inf (d(x))^{-\varpi}\left(\frac{u(x)}{A_{1} \phi(K(d(x)))}-1\right) \\
\lim _{d(x) \rightarrow 0} \sup (d(x))^{-\varpi}\left(\frac{u(x)}{A_{1} \phi(K(d(x)))}-1\right) \leq A_{3}+\varepsilon .
\end{gathered}
$$

Thus letting $\varepsilon \rightarrow 0$, we obtain (1.9). The proof is finished.

\section{Proof of Theorem 1.3}

In this section, we prove Theorem 1.3.

Let $0<a_{0}<1$ and

$$
w_{ \pm}=A_{1} \phi(K(d(x)))\left(1+\left(A_{4} \pm \varepsilon\right)(d(x))^{\varpi}\right), \quad x \in \Omega_{\delta_{1}} .
$$

By the Lagrange mean value theorem, we obtain that there exist $\lambda_{ \pm} \in(0,1)$ and

$$
\Phi_{ \pm}(d(x))=A_{1} \phi(K(d(x)))\left(1+\lambda_{ \pm}\left(A_{4} \pm \varepsilon\right)(d(x))^{\varpi}\right)
$$

such that, for $x \in \Omega_{\delta_{1}}$,

$$
f\left(w_{ \pm}(x)\right)=f\left(A_{1} \phi(K(d(x)))\right)+A_{1}\left(A_{4} \pm \varepsilon\right) \phi(K(d(x))) f^{\prime}\left(\Phi_{ \pm}(d(x))\right)(d(x))^{\varpi} .
$$

Since $f \in \mathrm{NRV}_{p}$, by Proposition 2.1 we obtain

$$
\lim _{d(x) \rightarrow 0} \frac{f\left(A_{1} \phi(K(d(x)))\right)}{f\left(\Phi_{ \pm}(d(x))\right)}=\lim _{d(x) \rightarrow 0} \frac{f^{\prime}\left(A_{1} \phi(K(d(x)))\right)}{f^{\prime}\left(\Phi_{ \pm}(d(x))\right)}=1 .
$$

Define $r=d(x)$ and

$$
\begin{aligned}
I_{1}(r)= & r^{-\varpi}(p-1)\left(1+\frac{\phi^{\prime}(K(r)) k^{\prime}(r)}{\phi^{\prime \prime}(K(r)) k^{2}(r)}-\frac{f\left(A_{1} \phi(K(r))\right)}{A_{1}^{p-1} f(\phi(K(r)))}\right) \\
I_{2 \pm}(r)= & \left(A_{4} \pm \varepsilon\right)(p-1)\left(p-1+\frac{\phi^{\prime}(K(r)) k^{\prime}(r)}{\phi^{\prime \prime}(K(r)) k^{2}(r)}+2 \varpi \frac{\phi^{\prime}(K(r))}{\phi^{\prime \prime}(K(r)) k(r) r}\right. \\
& +\frac{\varpi(\varpi-1) \phi(K(r))}{\phi^{\prime \prime}(K(r)) k^{2}(r) r^{2}} \\
& -A_{1} \frac{f^{\prime}\left(\Phi_{ \pm}(r)\right)}{f^{\prime}\left(A_{1} \phi(K(r))\right)} \frac{\phi(K(r)) f^{\prime}\left(A_{1} \phi(K(r))\right)}{A_{1}^{p-1} f(\phi(K(r)))} \\
& +(p-2)\left(\frac{\varpi \phi(K(r))}{\phi^{\prime}(K(r)) k(r) r}+\frac{\phi^{\prime}(K(r)) k^{\prime}(r)}{\phi^{\prime \prime}(K(r)) k^{2}(r)}\right. \\
& \left.\left.+\varpi \frac{\phi(K(r))}{\phi^{\prime}(K(r)) k(r)} \frac{\phi^{\prime}(K(r)) k^{\prime}(r)}{\phi^{\prime \prime}(K(r)) k^{2}(r) r}\right)+o\left(s^{\varpi}\right)\right)
\end{aligned}
$$




$$
\begin{aligned}
& I_{3 \pm}(x)=\Delta d(x)\left(\frac{\phi^{\prime}(K(r))}{\phi^{\prime \prime}(K(r)) k(r)}\left(r^{-\varpi}+\left(A_{4} \pm \varepsilon\right)\right)+\varpi r^{-1}\left(A_{4} \pm \varepsilon\right) \frac{\phi(K(r))}{\phi^{\prime \prime}(K(r)) k^{2}(r)}\right) \\
& I_{4 \pm}(r)=\left(c \mp a_{0} \varepsilon\right)(p-1) r^{\theta}\left(\frac{f\left(A_{1} \phi(K(r))\right)}{A_{1}^{p-1} f(\phi(K(r)))} r^{-\varpi}-\left(A_{4} \pm \varepsilon\right) \frac{f^{\prime}\left(\Phi_{ \pm}(r)\right) \phi(K(r))}{A_{1}^{p-1} f(\phi(K(r)))}\right) .
\end{aligned}
$$

By Lemmas 3.1, 3.6, and 3.9, combining with the choices of $A_{1}, A_{4}$, $\xi_{2}$ in Theorem 1.3, we see the following.

Lemma 6.1 Under the hypotheses in Theorem 1.3,

(i) $\lim _{r \rightarrow 0} I_{1}(r)=\lambda_{2}$, where

$$
\lambda_{2}= \begin{cases}\frac{(p-1)(\sigma+1-p)}{\sigma+1} D_{3 k} \text { Heaviside }(\theta-\varsigma):=\Theta_{2} & \text { if (i) or (ii) holds } \\ \Theta_{2}-q_{3} \xi_{2} p(p-1)\left(\frac{1}{(\sigma+1)(\sigma+1+\eta)}+\frac{\ln \frac{p}{p+1}}{(\sigma+1)(\sigma+1-p)}\right) & \text { if (iii) holds }\end{cases}
$$

(ii) $\lim _{r \rightarrow 0} I_{2 \pm}(r)=-\left(A_{4} \pm \varepsilon\right) \frac{p(p-1)(\sigma+1-p)}{\sigma+1}$;

(iii) $\lim _{d(x) \rightarrow 0} I_{3 \pm}(x)=0$;

(iv) $\lim _{r \rightarrow 0} I_{4 \pm}(r)=\frac{p(p-1)}{\sigma+1}\left(c \mp a_{0} \varepsilon\right) \operatorname{Heaviside}(\varsigma-\theta)$;

(v) $\lim _{d(x) \rightarrow 0}\left(I_{1}(r)+I_{2 \pm}(r)+I_{3 \pm}(x)+I_{4 \pm}(r)\right)= \pm \varepsilon \frac{p(p-1)}{\sigma+1}\left(\sigma+1-p+a_{0} \operatorname{Heaviside}(\varsigma-\theta)\right)$.

Proof of Theorem 1.3 Using a proof similar to that for Theorem 1.1, let

$$
\bar{u}_{\varepsilon}=A_{1} \phi\left(K\left(d_{1}(x)\right)\right)\left(1+\left(A_{4}+\varepsilon\right)\left(d_{1}(x)\right)^{\varpi}\right), \quad x \in D_{\sigma}^{-} .
$$

Then, by a direct calculation, we have for $x \in D_{\sigma}^{-}$

$$
\begin{aligned}
& \Delta \bar{u}_{\varepsilon}(x)-k^{p}\left(d_{1}(x)\right)\left(1+\left(c-a_{0} \varepsilon\right)\left(d_{1}(x)\right)^{\theta}\right) f\left(\bar{u}_{\varepsilon}(x)\right) \\
& \quad=A_{1}^{p-1}\left|\phi^{\prime}(K(r))\right|^{p-2} \phi^{\prime \prime}(K(r)) k^{p}(r) r^{\varpi}\left(I_{1}(r)+I_{2+}(r)+I_{3+}(x)+I_{4+}(r)\right) \\
& \quad \leq 0
\end{aligned}
$$

where $r=d_{1}(x)$, i.e., $\bar{u}_{\varepsilon}$ is a supersolution of equation (1.1) in $D_{\sigma}^{-}$.

In a similar way, we can show that

$$
\underline{u}_{\varepsilon}=A_{1} \phi\left(K\left(d_{2}(x)\right)\right)\left(1+\left(A_{4}-\varepsilon\right)\left(d_{2}(x)\right)^{\varpi}\right), \quad x \in D_{\sigma}^{+},
$$

is a subsolution of equation (1.1) in $D_{\sigma}^{+}$.

Using a proof similar to that for Theorem 1.1 , we can obtain, for $x \in \Omega_{2 \delta_{1 \varepsilon}}$,

$$
A_{4}-\varepsilon-\frac{M(d(x))^{-\varpi}}{A_{1} \phi(K(d(x)))} \leq(d(x))^{-\varpi}\left(\frac{u(x)}{A_{1} \phi(K(d(x)))}-1\right)
$$

and

$$
(d(x))^{-\varpi}\left(\frac{u(x)}{A_{1} \phi(K(d(x)))}-1\right) \leq A_{4}+\varepsilon+\frac{M(d(x))^{-\varpi}}{A_{1} \phi(K(d(x)))} .
$$

Consequently, by Lemma 3.6 and $0<\varpi<1$,

$$
A_{4}-\varepsilon \leq \lim _{d(x) \rightarrow 0} \inf (d(x))^{-\varpi}\left(\frac{u(x)}{A_{1} \phi(K(d(x)))}-1\right)
$$




$$
\lim _{d(x) \rightarrow 0} \sup (d(x))^{-\varpi}\left(\frac{u(x)}{A_{1} \phi(K(d(x)))}-1\right) \leq A_{4}+\varepsilon
$$

Thus letting $\varepsilon \rightarrow 0$, we obtain (1.9). The proof is finished.

\section{Acknowledgements}

The author expresses the sincere gratitude to the editors and referees for the careful reading of the original manuscript and useful comments that helped to improve the presentation of the results and accentuate important details.

\section{Funding}

This work was partially supported by NSF of China (Grant no. 11771196).

\section{Availability of data and materials}

Data sharing not applicable to this article as no datasets were generated or analysed during the current study.

\section{Competing interests}

The author declares that they have no competing interests.

\section{Authors' contributions}

All authors contributed equally to this work. They all read and approved the final version of the manuscript.

\section{Publisher's Note}

Springer Nature remains neutral with regard to jurisdictional claims in published maps and institutional affiliations.

\section{Received: 5 December 2018 Accepted: 26 March 2019 Published online: 03 April 2019}

\section{References}

1. Repovš, D.: Asymptotics for singular solutions of quasilinear elliptic equations with an absorption term. J. Math. Anal. Appl. 395, 78-85 (2012)

2. Bieberbach, L.: $\Delta u=e^{u}$ und die automorphen Funktionen. Math. Ann. 77, 173-212 (1916)

3. Keller, J.B.: On solutions of $\Delta u=f(u)$. Commun. Pure Appl. Math. 10, 503-510 (1957)

4. Osserman, R.: On the inequality $\Delta u \geq f(u)$. Pac. J. Math. 7, 1641-1647 (1957)

5. Loewner, C., Nirenberg, L.: Partial differential equations invariant under conformal or projective transformations. In: Contributions to Analysis (a Collection of Papers Dedicated to Lipman Bers), pp. 245-272. Academic Press, New York (1974)

6. Bandle, C., Marcus, M.: Large solutions of semilinear elliptic equations: existence, uniqueness and asymptotic behavior. J. Anal. Math. 58, 9-24 (1992)

7. Cîrstea, F., Rădulescu, V.: Uniqueness of the blow-up boundary solution of logistic equations with absorbtion. C. R. Acad. Sci. Paris, Ser. I 335, 447-452 (2002)

8. Cîrstea, F., Rădulescu, V.: Nonlinear problems with boundary blow-up: a Karamata regular variation theory approach. Asymptot. Anal. 46, 275-298 (2006)

9. Cîrstea, F., Rădulescu, V.: Blow-up solutions for semilinear elliptic problems. Nonlinear Anal. 48, 541-554 (2002)

10. Cirstea, F., Du, Y.: General uniqueness results and variation speed for blow-up solutions of elliptic equations. Proc. Lond. Math. Soc. 91, 459-482 (2005)

11. Zhang, Z., Ma, Y., Mi, L., Li, X.: Blow-up rates of large solutions for elliptic equations. J. Differ. Equ. 249, 180-199 (2010)

12. Zhang, Z:: The second expansion of large solutions for semilinear elliptic equations. Nonlinear Anal. 74, 3445-3457 (2011)

13. Huang, S., Tian, Q.: Second-order estimate for blow-up solutions of elliptic equations. Nonlinear Anal. 74, 2031-2044 (2011)

14. Huang, S., Tian, Q., Zhang, S., Xi, J.: A second order estimate for blow-up solutions of elliptic equations. Nonlinear Anal. $74,2342-2350(2011)$

15. Mi, L., Liu, B.: Second order expansion for blowup solutions of semilinear elliptic problems. Nonlinear Anal. 75 $2591-2613(2012)$

16. Gladiali, F., Porru, G.: Estimates for explosive solutions to $p$-Laplace equations. In: Progress in Partial Differential Equations, Pont-á-Mousson, 1997, vol. 1; in: Pitman Res. Notes Math. Ser., vol. 383, Longman, Harlow, 1998, pp. $117-127$

17. Mohammed, A.: Existence and asymptotic behavior of blow-up solutions to weighted quasilinear equations. J. Math. Anal. Appl. 298, 621-637 (2004)

18. Mohammed, A.: Boundary asymptotic and uniqueness of solutions to the $p$-Laplacian with infinite boundary values. J. Math. Anal. Appl. 325, 480-489 (2007)

19. Covei, D.: Large and entire large solution for a quasilinear problem. Nonlinear Anal. 70, 1738-1745 (2009)

20. García-Melián, J., Rossi, J.D., Sabina, J.: Large solutions to the $p$-Laplacian for large p. Calc. Var. Partial Differ. Equ. 31 187-204 (2008)

21. García-Melián, J.: Large solutions for equations involving the $p$-Laplacian and singular weights. Z. Angew. Math. Phys. 129, 1-4 (2008)

22. Huang, P., Shieh, M., Wang, S.: Explicit necessary and sufficient conditions for the existence of nonnegative solutions of a $p$-Laplacian blow-up problem. Taiwan. J. Math. 13, 1077-1093 (2009)

23. Wei, L., Zhu, J.: The existence and blow-up rate of large solutions of one-dimensional $p$-Laplacian equations. Nonlinear Anal., Real World Appl. 13, 665-676 (2012) 
24. Zhang, Z: Large solutions to the Monge-Ampère equations with nonlinear gradient terms: existence and boundary behavior. J. Differ. Equ. 264, 263-296 (2018)

25. Zhang, Z: Boundary behavior of large solutions to $p$-Laplacian elliptic equations. Nonlinear Anal., Real World Appl. 33, 40-57 (2017)

26. Zhang, X., Du, Y.: Sharp conditions for the existence of boundary blow-up solutions to the Monge-Ampère equation. Calc. Var. Partial Differ. Equ. 57(2), 30 (2018)

27. Zhang, X., Feng, M.: Boundary blow-up solutions to the $k$-Hessian equation with a weakly superlinear nonlinearity. J. Math. Anal. Appl. 464, 456-472 (2018)

28. Zhang, X., Feng, M.: Boundary blow-up solutions to the $k$-Hessian equation with singular weights. Nonlinear Anal. $167,51-66(2018)$

29. $\mathrm{Mi}, \mathrm{L} ., \mathrm{Qi}, \mathrm{Y}$., Liu, B.: Blow-up rate of the unique solution for a class of one-dimensional $p$-Laplacian equations. Nonlinear Anal., Real World Appl. 13, 2734-2746 (2012)

30. Seneta, R.: Regular Varying Functions. Lecture Notes in Math., vol. 508. Springer, Berlin (1976)

31. Resnick, S.I.: Extreme Values, Regular Variation, and Point Processes. Springer, New York (1987)

32. Maric, V.: Regular Variation and Differential Equations. Lecture Notes in Math., vol. 1726. Springer, Berlin (2000)

33. Tolksdorf, P.: On the Dirichlet problem for quasilinear equations in domains with conical boundary points. Commun. Partial Differ. Equ. 8, 773-817 (1983)

\section{Submit your manuscript to a SpringerOpen ${ }^{\circ}$ journal and benefit from:}

- Convenient online submission

- Rigorous peer review

- Open access: articles freely available online

- High visibility within the field

- Retaining the copyright to your article

Submit your next manuscript at $\boldsymbol{\nabla}$ springeropen.com 